\title{
Stability and Uniqueness of Global Solutions to Euler Equations with Exothermic Reaction
}

\author{
Kai $\mathrm{Hu}^{1}$ \\ School of Mathematics and Statistics, Southwest University, Chongqing, 400715, China
}

\begin{abstract}
We consider the Cauchy problems of a non-strictly hyperbolic system which describes the compressible Euler fluid with exothermic reaction. In this paper a Lyapunov-type functional is constructed for balance laws. By analysis of the flow generated by front tracking method, we prove the well-posedness theorems and present the local features of global solutions.
\end{abstract}

Keywords: Euler equations, Well-posedness, Front tracking method 2010 MSC: 35L45, 35L60, 35Q31, 35Q35

\section{Introduction}

We are concerned about the Cauchy problem of the exothermically reacting compressible Euler equations :

$$
\begin{aligned}
& v_{t}-u_{x}=0, \\
& u_{t}+p_{x}=0, \\
& E_{t}+(p u)_{x}=q Y \phi(T), \\
& Y_{t}=-Y \phi(T),
\end{aligned}
$$

where $v, u, T, p$ and $E$ respectively represent specific volume, velocity, temperature, pressure and total energy of compressible fluid. This system arises from

This paper is supported by National Natural Science Foundation of China (Grant No. 11426185), Fundamental Research Funds for the Central Universities (XDJK2014C075 and SWU113062) and China Scholarship Council.

${ }^{1}$ E-mail address: hukaimath@swu.edu.cn

Preprint submitted to .

October 25, 2018 
combustion theory in continuum physics. Constant $q>0$ denotes unit binding energy released by combustion. $Y$ represents the mass fraction of reactant in mixed fluid, where $0 \leq Y \leq 1$. Assume that adiabatic constant $\gamma$ and specific heat constant $c$ of the mixture are independent of $Y$. The procedure of releaseing chemical energy is controlled by reaction-rate function $\phi(T)$, which is a $C^{1}$ function w.r.t. $T$. Suppose that $\phi(T)$ is positive and increasing on $[0,+\infty)$. For instance it is of Arrhenius form $\phi(T)=T^{\alpha} \mathrm{e}^{-\beta / T}$ with constants $\alpha, \beta>0$. In present paper, we prescribe the initial data

$$
U_{0}(x)=\left(v_{0}(x), u_{0}(x), E_{0}(x), Y_{0}(x)\right), \quad x \in \mathbb{R}
$$

with $Y_{0}(x) \in L^{1}(\mathbb{R})$ and variation $T . V .\left(U_{0}\right)<+\infty$.

System (1) is a typical hyperbolic balance law. It can be formulated by

$$
U_{t}+F(U)_{x}=G(U)
$$

where $U=(v, u, E, Y)$. Well-posedness of solutions to balance laws was already established under particular circumstances. See [1, 2, 3, 4, 5, 6] and the references therein. Generally speaking, strictly hyperbolic balance laws has local weak solutions with bounded variation. Their variation may blow up within finite time due to damping effect. Therefore, in order to extend them to global solutions, one must impose appropriate dissipative conditions on source $G$. Specifically let matrix

$$
\tilde{G}=R^{-1}(\mathbf{0}) \mathrm{d} G(\mathbf{0}) R(\mathbf{0})
$$

where matrix $R(U)$ consists of linearly independent right eigenvectors of $d F(U)$. Dafermos and Hsiao[1] observe that when $\tilde{G}$ is strictly diagonally dominant, system (3) admits a global BV solution, whose variation decreases exponentially as $t \rightarrow \infty$. Concerning non-autonomous source $G=G(x, U)$, Amadori and collaborators [2] proposed a non-resonance condition and a smallness condition on $\omega$,

$$
|G(\cdot, U)|+\left\|\nabla_{\mathbf{u}} G(\cdot, U)\right\| \leq \omega \in L^{1} \cap L^{\infty}(\mathbb{R})
$$


where $\|\cdot\|$ denotes the Euclidian norm. Such conditions imply global wellposedness. Whereas our system (11) is ruled out of previous situations in early research, because it is not strictly hyperbolic. Two eigenvalues of Jacobian $d F(U)$ completely coincide. It is known that resonance of different fields possibly leads to loss of uniqueness of entropy solutions. Although one reduce (11) to $3 \times 3$ strictly hyperbolic system by separating unknown $Y$ from equations (1), the diagonal dominance condition still fails to hold. So well-posedness of our system indeed possesses new difficulties that differ from the previous works.

The Cauchy problem (1) (2) has been intensively discussed in recent research. Main difficulty of system (11) is governing the damping induced by term $Y \phi(T)$, which arises from exothermical reaction and amplifies any oscillation. To achieve this, 7] demands initial temperature satisfies

$$
\phi\left(T_{0}(x)\right)>\phi>0, \quad \forall x \in \mathbb{R},
$$

with some constant $\phi$. Then existence of global entropy solutions was verified in [7, 8] if $U_{0}$ is of small total variation. Related results on Navier-Stokes equations in combustion are referred to [9, 10, 11, 12]. However, uniqueness and stability of global solutions remain unknown. Our paper is intended to give the complete conclusions on this problem.

In this paper, we consider the global solution $U(t, x)$ constructed by fractional step wave front tracking scheme, which determines a semigroup trajectory. On stability issue, we must refer the pioneering work by Bressan, Liu and Yang (cf. [13, 14]). They devised an elegant functional $\Phi$ for conservation laws to analyze the $L^{1}$ distance of distinct trajectories. Unfortunately this functional is invalid for system (11), since the exothermical damping removes the rough monotonicity of $\Phi$. It is tough to construct an appropriate functional instead of $\Phi$. Inspired by the argument in [13], we improve the weight included in Lyapunov functional, and then establish the stability theorem for system (11) as follows.

Theorem 1. Assume that T.V. $\left(U_{0}\right)$ and $\left\|Y_{0}\right\|_{\infty}$ are sufficiently small, moreover, condition (4) holds. Then according to fractional step wave front tracking scheme, there exists a family of domains $\left\{\mathcal{D}_{t}\right\}_{t \geq 0}$, a unique semigroup 
$\mathcal{P}:[0,+\infty) \times \mathcal{D}_{\infty} \rightarrow \mathcal{D}_{\infty}$ and a constant $L>0$ such that

(i) $U(t, x) \doteq \mathcal{P}_{t}\left(U_{0}\right)$ with $U_{0} \in \mathcal{D}_{0}$ is an entropy weak solution to problem (1) (2));

(ii) for any $U_{0}, V_{0} \in \mathcal{D}_{0}$ and $t, s \geq 0$, there holds

$$
\begin{aligned}
& \mathcal{P}_{0}\left(U_{0}\right)=U_{0}, \quad \mathcal{P}_{t}\left(\mathcal{P}_{s}\left(U_{0}\right)\right)=\mathcal{P}_{s+t}\left(U_{0}\right), \\
& \left\|\mathcal{P}_{t}\left(U_{0}\right)-\mathcal{P}_{s}\left(V_{0}\right)\right\|_{L^{1}} \leq L\left(\left\|U_{0}-V_{0}\right\|_{L^{1}}+|t-s|\right) .
\end{aligned}
$$

Remark 1. We construct domain $\mathcal{D}_{t}$ by $(17)$ in a general setting. Classic stability theory regards the constant state, especially $U \equiv \mathbf{0}$, as a equilibrium point for balance laws. However, condition (4) in this paper causes the temperature is globally away from 0 . We thereby replace the equilibrium state with a Riemann data $U_{\infty}$ (see Subsection 2.2), which may connect two distinct states at $x= \pm \infty$. Theorem 1 demonstrates that the flow close to $U_{\infty}$ is still stable under small perturbation in $B V \cap L^{1}$ space.

Furthermore, we find that the local characteristics of solution $U(t, x)$ are completely determined by either conservation laws (43) or transport equations (44). To illustrate this, we introduce an entropy solution $U_{\xi}^{C}(t, x)$ to (43) and a solution $U_{\xi}^{T}(t, x)$ to (44), then derive the following theorem.

Theorem 2. Assume that T.V. $\left(U_{0}\right)$ and $\left\|Y_{0}\right\|_{\infty}$ are sufficiently small, moreover, condition (4) holds. Let $\mathcal{P}$ be a semigroup defined in Theorem 1, and $\hat{\lambda}$ be a positive constant larger than all characteristic speeds of system (1). If $U(t, x)=\mathcal{P}_{t}\left(U_{0}\right)$ with $U_{0} \in \mathcal{D}_{0}$, then for every $s \geq 0$ and $a<\xi<b$, there holds

$$
\begin{aligned}
& \lim _{\theta \rightarrow 0^{+}} \frac{1}{\theta} \int_{\xi-\theta \hat{\lambda}}^{\xi+\theta \hat{\lambda}}\left\|U(s+\theta, x)-U_{\xi}^{C}(\theta, x)\right\| d x=0, \\
& \varlimsup_{\theta \rightarrow 0^{+}} \frac{1}{\theta} \int_{a+\theta \hat{\lambda}}^{b-\theta \hat{\lambda}}\left\|U(s+\theta, x)-U_{\xi}^{T}(\theta, x)\right\| d x \leq C_{0}\{T . V .(U(s) ;(a, b))\}^{2},
\end{aligned}
$$

where $C_{0}$ is a positive constant. Conversely, if mapping $U:[0,+\infty) \rightarrow \mathcal{D}_{\infty}$ is Lipschitz continuous in $L^{1}$-topology and satisfies (7) (8) for almost every $s \geq 0$ and $a<\xi<b$, then $U(t)$ must coincide with the semigroup trajectory $\mathcal{P}_{t}\left(U_{0}\right)$.

Theorem 2 reveals that the entropy solution possessing local features (7) (8) is actually unique, no matter what approach we adopt. 


\section{Preliminary}

The author [8] developed the existence theorem for general mixed Euler fluid. First of all, we directly state the algorithm and the estimates of Glimm functional for system (1). As mentioned in Section 1, assume thermodynamics parameters $\gamma$ and $c$ remain constants throughout the paper.

\subsection{Fractional Step Wave-Front Tracking Scheme}

The strategy of this scheme is separating the effect of source term from original system, and then transforming it into a linear problem. Now we initiate the procedure by simplifying initial data $U_{0}$. For each $\varepsilon>0$, choose suitable $U_{0}^{\varepsilon}(x)$ as an approximation of $U_{0}(x)$ such that

- $U_{0}^{\varepsilon}(x)$ is piecewise constant, and has finite discontinuous points $\left\{x_{\alpha}\right\} \subset$ $[-1 / \varepsilon, 1 / \varepsilon]$.

- T.V. $\left(U_{0}^{\varepsilon}\right) \leq T . V .\left(U_{0}\right)$.

- $\int_{-\frac{1}{\varepsilon}}^{\frac{1}{\varepsilon}}\left\|U_{0}^{\varepsilon}(x)-U_{0}(x)\right\| d x<\varepsilon$.

Then divide time interval $[0,1 / \varepsilon]$ into $N$ subintervals, such that division points

$$
t_{0}=0, \quad t_{1}=\varepsilon, \quad t_{2}=2 \varepsilon, \quad \cdots \quad t_{N}=N \varepsilon .
$$

Additionally set $t_{N+1}=+\infty$.

In each strip domain $\Omega_{k}=\left\{(t, x) \mid t_{k} \leq t<t_{k+1}, \quad x \in \mathbb{R}\right\}(k=0,1, \cdots, N)$, we approximately solve the initial value problem of conservation law

$$
\begin{aligned}
& U_{t}+F(U)_{x}=0, \quad(t, x) \in \Omega_{k}, \\
& U\left(t_{k}, x\right)=U^{\varepsilon}\left(t_{k}, x\right)
\end{aligned}
$$

by standard wave-front tracking method. See [15, 16] for this method in detail. Define the solution to (9) by

$$
U^{\varepsilon}(t, x) \doteq \mathcal{S}_{t}^{\varepsilon}\left(U^{\varepsilon}\left(t_{k}, x\right)\right), \quad(t, x) \in \Omega_{k},
$$


where $\mathcal{S}_{t}^{\varepsilon}$ is the approximate solution operator of (9) . Notice that $Y(t, x) \equiv$ $Y\left(t_{k}, x\right)$ for every point $(t, x) \in \Omega_{k}$. When $t=t_{k}$, the chemical reaction will be activated. Therefore consider an ordinary differential equations,

$$
\begin{aligned}
& U_{t}=G(U), \quad t \in[0, \varepsilon], \\
& U(0, x)=U^{\varepsilon}\left(t_{k}-, x\right)
\end{aligned}
$$

where $U^{\varepsilon}\left(t_{k}-, x\right) \doteq \lim _{t \rightarrow t_{k}^{-}} U^{\varepsilon}(t, x)$. We define the linearly approximate solution by

$$
\mathcal{T}_{t}(U(0, x)) \doteq U(0, x)+G(U(0, x)) t
$$

where $\mathcal{T}_{t}$ denotes the approximate solution operator of (10). Then assign the value of $U^{\varepsilon}(t, x)$ after reaction by

$$
\begin{aligned}
U^{\varepsilon}\left(t_{k}, x\right) & \doteq \mathcal{T}_{\varepsilon}\left(U^{\varepsilon}\left(t_{k}-, x\right)\right) \\
& =U^{\varepsilon}\left(t_{k}-, x\right)+G\left(U^{\varepsilon}\left(t_{k}-, x\right)\right) \varepsilon
\end{aligned}
$$

In summary, we formulate the $\varepsilon$-approximate solution to system (11) as follow,

$$
U^{\varepsilon}(t, x)=\mathcal{P}_{t}^{\varepsilon}\left(U_{0}^{\varepsilon}(x)\right) \doteq \mathcal{S}_{t-k \varepsilon}^{\varepsilon}\left(\mathcal{T}_{\varepsilon} \mathcal{S}_{\varepsilon}^{\varepsilon}\right)^{k}\left(U_{0}^{\varepsilon}(x)\right),
$$

where integer $k=\min \{[t / \varepsilon], N\}$. $\mathcal{P}_{t}^{\varepsilon}$ is actually a composition of mappings $\mathcal{T}_{\varepsilon}$, $\mathcal{S}_{\varepsilon}^{\varepsilon}$, etc.. It was proved in [8] that as $\varepsilon \rightarrow 0$, the sequence of $U^{\varepsilon}(t, x)$ converges to an entropy weak solution $U(t, x)=\mathcal{P}_{t}\left(U_{0}\right)$ of system (11).

\subsection{Evolution of Approximate Solutions in $\mathcal{D}_{\infty}$}

In this subsection we will explain how the flow of $\mathcal{P}_{t}^{\varepsilon}\left(U_{0}^{\varepsilon}(x)\right)$ evolves as $t$ increases. It is necessary to understand some fundamental properties of system (11) in advance. The Jacobian $d F(U)$ has four eigenvalues, i.e.

$$
\lambda_{1}=-\sqrt{-\gamma p_{v}}, \quad \lambda_{2}=0, \quad \lambda_{3}=\sqrt{-\gamma p_{v}}, \quad \lambda_{4}=0,
$$

where $p=p(v, u, E)$. So we say that system (11) is not strictly hyperbolic. The waves of the first three fields, called elementary waves, contain shock, rarefaction wave and contact discontinuity; the wave of the 4th field, called $Y$-wave, is of speed $\lambda_{4}=0$. To $\varepsilon$-approximate solutions, we add a family of non-physical 
waves (abbr. $N P$ ) moving at fixed speed $\hat{\lambda}$. By construction, total strength of $N P$ waves is less than $\varepsilon$. See [8, 15, 16].

Next introduce some necessary functionals. They are developed from the classic functionals in [7, 15]. For each $U(t, x)$, let functional

$$
\mathcal{V}(U(t)) \doteq \sum(|\alpha|+|\sigma|+M|\delta|)
$$

where $|\alpha|,|\sigma|$ and $|\delta|$ respectively denote the strengths of elementary waves, NP wave and $Y$-wave in $U(t)$. Positive constant $M$ is used to remove bad terms arising from estimates of Glimm functional; see Lemma 8 in [8]. In order to distinguish $Y$-waves which locate in different positions at time $t$, we write $\left|\delta_{\alpha}\right|$ with subscript $\alpha$. It means that this $Y$-wave front of strength $\left|\delta_{\alpha}\right|$ intersects with a front of strength $|\alpha|$ at some point $(\bar{t}, \bar{x})$, where $\bar{t} \leq t$. We define $\left|\delta_{\beta}\right|$, $\left|\delta_{\sigma}\right|,\left|\bar{\delta}_{\alpha}\right|,\left|\bar{\delta}_{\beta}\right|$ etc. in analogous way. Furthermore, write

$$
\begin{aligned}
Q(U(t)) \doteq & \sum_{\mathrm{App}}|\alpha| \cdot|\beta|+\sum_{\mathrm{App}}|\alpha| \cdot M\left|\delta_{\beta}\right|+\sum M\left|\delta_{\alpha}\right| \cdot M\left|\delta_{\beta}\right| \\
& +\sum_{\mathrm{App}}|\sigma| \cdot|\beta|+\sum_{\mathrm{App}}|\sigma| \cdot M\left|\delta_{\beta}\right|+\sum M\left|\delta_{\sigma}\right| \cdot M\left|\delta_{\beta}\right|
\end{aligned}
$$

to measure the interaction of any two approaching waves except that of $N P$ wave and $Y$-wave. We require the term $M\left|\delta_{\alpha}\right| \cdot M\left|\delta_{\beta}\right|$ is included in $Q(U(t))$ if corresponding $|\alpha| \cdot|\beta|$ exists in $\sum_{\mathrm{App}}|\alpha| \cdot|\beta|$. Analogously whether $M\left|\delta_{\sigma}\right| \cdot M\left|\delta_{\beta}\right|$ is included in $Q(U(t))$ depends on $|\sigma| \cdot|\beta|$.

Define modified Glimm functional

$$
\mathcal{F}(U(t)) \doteq \mathcal{V}(U(t))+C \cdot Q(U(t))
$$

where positive constant $C$ is so large that estimate (15) holds. To generate a Lipschitz continuous semigroup, we require that $Y_{0} \in L^{1} \cap L^{\infty}(\mathbb{R})$. However, $\left\|Y_{0}\right\|_{L^{1}}$ may be arbitrarily large.

Suppose there exist constants $\phi$ and $\psi$ such that $0<\phi<\phi\left(T_{0}\right)<\psi$. Thus it is reasonable to assert that

$$
0<\phi \leq \phi(T(t, x)) \leq \psi
$$


holds for all $t>0$ provided $U(t, x)$ is sufficiently close to initial $U_{0}(x)$ in $L^{\infty}(\mathbb{R})$. It was proved that the Glimm functional $\mathcal{F}$ designed for system (10) is possibly increasing but eventually bounded, provided initial data is suitably small. Here we directly state the following estimates of $\mathcal{F}$ in Lemma 1 . Also see conclusions $(41)(42)$ in [8].

Lemma 1. Assume that T.V. $\left(U_{0}\right)$ and $\left\|Y_{0}\right\|_{\infty}$ are small enough, moreover, condition (4) holds. Let $U^{\varepsilon}$ be an approximate solution defined in (12), and integer $k=[t / \varepsilon]$. Then

$$
\begin{aligned}
\mathcal{F}\left(U^{\varepsilon}(t)\right) & \leq \mathcal{F}\left(U^{\varepsilon}\left(t_{k}-\right)\right) \exp \left\{B\left\|Y_{0}\right\|_{\infty} \mathrm{e}^{-\phi k \varepsilon} \varepsilon\right\} \\
& \leq \mathcal{F}\left(U_{0}\right) \exp \left\{B\left\|Y_{0}\right\|_{\infty} \sum_{i \varepsilon \leq t} \mathrm{e}^{-\phi i \varepsilon} \varepsilon\right\} \\
& \leq \mathcal{F}\left(U_{0}\right) \exp \left\{\frac{B\left\|Y_{0}\right\|_{\infty}}{\phi}\right\} \\
& \leq \overline{\mathcal{F}} \\
Y(t) & \leq Y(0) \mathrm{e}^{-\phi k \varepsilon}
\end{aligned}
$$

for small $\varepsilon>0$, where $i \in \mathbb{N}_{+}$, both $B$ and $\overline{\mathcal{F}}$ are constants .

Comparing (15) with original result in [8], we only maintain the first order terms of $\varepsilon$, because all higher order ones can be absorbed if $\varepsilon$ is small. Since $\mathcal{F}(U)$ is equivalent to T.V. $(U)$, there exists a constant $B^{*}>0$ such that

$$
\mathcal{F}\left(U_{0}\right)<\epsilon \quad \text { implies } \quad\left\|Y_{0}\right\|_{\infty}<B^{*} \epsilon
$$

for each $\epsilon>0$. Given $\epsilon$, define

$$
\begin{aligned}
& \epsilon(t) \doteq \epsilon \cdot \exp \left\{B B^{*} \epsilon \int_{0}^{t} \mathrm{e}^{-\phi s} \mathrm{~d} s\right\}=\epsilon \cdot \exp \left\{\frac{B B^{*} \epsilon}{\phi}\left(1-\mathrm{e}^{-\phi t}\right)\right\}, \\
& \epsilon_{\infty} \doteq \epsilon \cdot \exp \left\{\frac{B B^{*} \epsilon}{\phi}\right\} .
\end{aligned}
$$

Set vector $\mathcal{U} \doteq(v, u, E)$. State $U_{\infty}=\left(\mathcal{U}_{\infty}(x), 0\right)$ denotes a Riemann initial data with two pieces of constant states, i.e.

$$
\mathcal{U}_{\infty}(x)= \begin{cases}\mathcal{U}_{r}, & x>0 \\ \mathcal{U}_{l}, & x<0\end{cases}
$$


Evidentally $G\left(U_{\infty}\right)=\mathbf{0}$ in (11) and (3). Now we can explicitly define the domains where the flow $\mathcal{P}_{t}^{\varepsilon}(U)$ evolves. In fact, for every $t \geq 0$, set

$$
\begin{array}{r}
\mathcal{M}_{t} \doteq \mathbf{c l}\left\{\bar{U} \in L^{1}\left(\mathbb{R} ; \mathbb{R}^{4}\right) \mid \bar{U}\right. \text { is piecewise constant, } \\
\left.\mathcal{F}\left(\bar{U}+U_{\infty}\right)<\epsilon(t),\|\bar{Y}\|_{\infty}<B^{*} \epsilon \mathrm{e}^{-\phi t}\right\}, \\
\mathcal{M}_{\infty} \doteq \mathbf{c l}\left\{\bar{U} \in L^{1}\left(\mathbb{R} ; \mathbb{R}^{4}\right) \mid \bar{U}\right. \text { is piecewise constant, } \\
\left.\mathcal{F}\left(\bar{U}+U_{\infty}\right)<\epsilon_{\infty},\|\bar{Y}\|_{\infty}=0\right\},
\end{array}
$$

where $\mathbf{c l}$ denotes closure in $L^{1}$ topology. Then define domain

$$
\mathcal{D}_{t} \doteq\left\{U \mid U=\bar{U}+U_{\infty}, \bar{U} \in \bigcup_{s \leq t} \mathcal{M}_{s}\right\} .
$$

These domains obviously satisfy $\mathcal{D}_{s} \subset \mathcal{D}_{t} \subset \mathcal{D}_{\infty}$ for $0 \leq s<t$.

We complete the section by the dynamics of $\mathcal{P}_{t}^{\varepsilon}(U)$ in $\mathcal{D}_{\infty}$.

Lemma 2. Assume that $\epsilon$ is sufficiently small. If $U \in \mathcal{D}_{s}$ is a piecewise constant function, then there exists a positive $\bar{t}$, which depends on $U, \epsilon$ and $s$, satisfying that

(1) $\mathcal{T}_{t}(U) \in \mathcal{D}_{s+t}$ for $t \in(0, \bar{t})$;

(2) $\mathcal{S}_{t}^{\varepsilon}(U) \in \mathcal{D}_{s}, \mathcal{P}_{t}^{\varepsilon}(U) \in \mathcal{D}_{s+t}$ for any $t \in(0,+\infty)$, provided $\varepsilon \ll \bar{t}$.

Proof 1. If $U \in \mathcal{D}_{s}$, there exists some $r<s$ such that $U \in \mathcal{M}_{r}$. This gives

$$
\begin{aligned}
& \mathcal{F}(U)<\epsilon(r)=\epsilon \cdot \exp \left\{\frac{B B^{*} \epsilon}{\phi}\left(1-\mathrm{e}^{-\phi r}\right)\right\}, \\
& \|Y\|_{\infty}<B^{*} \epsilon \mathrm{e}^{-\phi r} .
\end{aligned}
$$

Thanks to Lemma 10 in [8] and the definition of $\mathcal{T}_{t}$, we have the following estimates.

$$
\begin{gathered}
\mathcal{F}\left(\mathcal{T}_{t}(U)\right) \leq \mathcal{F}(U) \exp \left\{B\|Y\|_{\infty} \mathrm{e}^{-\phi t} t\right\} \\
\leq \mathcal{F}(U) \exp \left\{B\|Y\|_{\infty} t\right\} \\
\mathcal{T}_{t} Y=Y-t Y \phi(T) \leq Y \mathrm{e}^{-\phi t},
\end{gathered}
$$


where $\mathcal{T}_{t} Y$ denotes the $Y$-component of $\mathcal{T}_{t}(U)$. Immediately, they imply

$$
\begin{aligned}
& \mathcal{F}\left(\mathcal{T}_{t}(U)\right) \leq \epsilon \cdot \exp \left\{\frac{B B^{*} \epsilon}{\phi}\left(1-\mathrm{e}^{-\phi r}\right)+B\|Y\|_{\infty} t\right\}, \\
& \left\|\mathcal{T}_{t} Y\right\|_{\infty} \leq\|Y\|_{\infty} \mathrm{e}^{-\phi t}<B^{*} \epsilon \mathrm{e}^{-\phi(r+t)} \quad \text { if } t \phi<1 .
\end{aligned}
$$

Set function

$$
\varphi(t)=\frac{\phi t}{1-\mathrm{e}^{-\phi t}} .
$$

Since $\varphi(0) \rightarrow 1$ as $t \rightarrow 0$, we select a positive $\bar{t}$ such that

$$
\begin{gathered}
\|Y\|_{\infty} \varphi(t)<B^{*} \epsilon \mathrm{e}^{-\phi r}, \\
\text { i.e. } \quad B\|Y\|_{\infty} t<\frac{B B^{*} \epsilon}{\phi} \mathrm{e}^{-\phi r}\left(1-\mathrm{e}^{-\phi t}\right)
\end{gathered}
$$

for every $t \in(0, \bar{t})$. Thus it follows from (19) that

$$
\mathcal{F}\left(\mathcal{T}_{t}(U)\right)<\epsilon(r) \exp \left\{B\|Y\|_{\infty} t\right\}<\epsilon \cdot \exp \left\{\frac{B B^{*} \epsilon}{\phi}\left(1-\mathrm{e}^{-\phi(r+t)}\right)\right\} .
$$

(20) and (21) guarantee $\mathcal{T}_{t}(U) \in \mathcal{M}_{r+t} \subset \mathcal{D}_{s+t}$.

2. Since $0<\varepsilon \ll \bar{t}$, it is deduced from standard argument of front tracking scheme that the Glimm functional $\mathcal{F}\left(\mathcal{S}_{t}^{\varepsilon}(U)\right)$ is decreasing in $t$, provided T.V.(U) is small enough. Therefore, if $U \in \mathcal{D}_{s}$, the fact $\mathcal{F}\left(\mathcal{S}_{t}^{\varepsilon}(U)\right) \leq \mathcal{F}(U)$ and $Y(t) \equiv$ $Y(0)$ yields $\mathcal{S}_{t}^{\varepsilon}(U) \in \mathcal{D}_{s}$ for any $t>0$. Recall that $\mathcal{P}_{t}^{\varepsilon}(U)=\mathcal{S}_{t-k \varepsilon}^{\varepsilon}\left(\mathcal{T}_{\varepsilon} \mathcal{S}_{\varepsilon}^{\varepsilon}\right)^{k}(U)$ where $k=[t / \varepsilon]$. The preceding results of $\mathcal{T}_{t}$ and $\mathcal{S}_{t}$ directly yield $\mathcal{P}_{t}^{\varepsilon}(U) \in \mathcal{D}_{s+t}$.

\section{Stability of Entropy Solution}

We will construct a functional in this section to measure the distance of two approximate solutions. This functional is almost decreasing in time $t$, which implies the continuous dependence of entropy solutions on initial data.

\subsection{Lyapunov Functional}

Assume initial data $U_{0}$ and $V_{0}$ belong to $\mathcal{D}_{0} . U_{0}^{\varepsilon}$ and $V_{0}^{\varepsilon}$ are corresponding $\varepsilon$-approximations. Let

$$
\mathcal{P}_{t}^{\varepsilon}\left(U_{0}^{\varepsilon}\right)=U^{\varepsilon}(t, x)=\left(\mathcal{U}_{1}, Y_{1}\right)
$$




$$
\mathcal{P}_{t}^{\varepsilon}\left(V_{0}^{\varepsilon}\right)=V^{\varepsilon}(t, x)=\left(\mathcal{U}_{2}, Y_{2}\right) .
$$

Here every state $U$ (or $V$ ) is decomposed into components $\mathcal{U} \doteq(v, u, E)$ and $Y$.

Consider equations (11) in phase space. The $i$-th family of Hugoniot curves is determined by the mapping $H_{i}$. For given states $\mathcal{U}_{1}$ and $\mathcal{U}_{2}$, there exists unique vector $\mathbf{q}=\left(q_{1}, q_{2}, q_{3}\right)$ such that

$$
\mathcal{U}_{2}=H(\mathbf{q})\left(\mathcal{U}_{1}\right) \doteq H_{3}\left(q_{3}\right) \circ H_{2}\left(q_{2}\right) \circ H_{1}\left(q_{1}\right)\left(\mathcal{U}_{1}\right)
$$

at every point $(t, x)$. It is known that total strength $\sum_{i=1}^{3}\left|q_{i}\right|$ is equivalent to || $\mathcal{U}_{1}-\mathcal{U}_{2}||$

We introduce the Lyapunov functional

$$
\Phi\left(U^{\varepsilon}(t), V^{\varepsilon}(t)\right) \doteq \sum_{i=1}^{3} \int_{-\infty}^{+\infty}\left(\left|q_{i}(t, x)\right|+\kappa_{1}\left|Y_{1}(t, x)-Y_{2}(t, x)\right|\right) W_{i}(t, x) d x .
$$

This functional is developed from the one in [13]. It is worth pointing out that, in contrast with $\Phi$ in [13], we add the term of $Y$ and modify the weight $W_{i}$ in order to overcome the damping effect. Specifically, let $\alpha$ be a jump of the $k_{\alpha}$-th family $\left(1 \leq k_{\alpha} \leq 3\right)$, whose location is $\left(t, x_{\alpha}\right)$ and strength $|\alpha|$. Let $\delta$ be a jump of $Y$-wave, whose strength is $|\delta|$. Notation $\mathcal{J}(U)$ denotes a set of all jumps of $U$ for given time $t$. Set $\mathcal{J} \doteq \mathcal{J}\left(U^{\varepsilon}\right) \bigcup \mathcal{J}\left(V^{\varepsilon}\right)$. Then the weight

$$
W_{i}(t, x) \doteq 1+\kappa_{2} A_{i}(t, x)+\kappa_{3}\left\{Q\left(U^{\varepsilon}\right)+Q\left(V^{\varepsilon}\right)\right\}+\kappa_{4} B(t),
$$

where for $i=1,3$,

$$
\begin{aligned}
& A_{i}(t, x)=\left[\sum_{\substack{x_{\alpha}<x, i<k_{\alpha} \leq 3, \alpha \in \mathcal{J}}}+\sum_{x_{\alpha}>x, 1 \leq k_{\alpha}<i,}\right]|\alpha|+\sum_{\delta \in \mathcal{J}} M|\delta| \\
& +\left\{\begin{array}{l}
{\left[\sum_{\substack{k_{\alpha}=i, x_{\alpha}<x, \alpha \in \mathcal{J}\left(U^{\varepsilon}\right)}}+\sum_{\substack{k_{\alpha}=i, x_{\alpha}>x \\
\alpha \in \mathcal{J}\left(V^{\varepsilon}\right)}}\right]|\alpha| \quad \text { if } q_{i}(x)<0,} \\
{\left[\sum_{\substack{\sum_{\alpha}=i, x_{\alpha}<x, \alpha \in \mathcal{J}\left(V^{\varepsilon}\right)}}+\sum_{\substack{k_{\alpha}=i, x_{\alpha}>x \\
\alpha \in \mathcal{J}\left(U^{\varepsilon}\right)}}\right]|\alpha| \quad \text { if } q_{i}(x)>0,}
\end{array}\right.
\end{aligned}
$$




$$
\begin{aligned}
A_{2}(t, x) & =\left[\sum_{\substack{x_{\alpha}<x, k_{\alpha}=3, \alpha \in \mathcal{J}}}+\sum_{\substack{x_{\alpha}>x, k_{\alpha}=1, \alpha \in \mathcal{J}}}\right]|\alpha|+\sum_{\delta \in \mathcal{J}} M|\delta|, \\
B(t) & =\sum_{j \varepsilon>t}\left(\left\|Y_{1}(0, \cdot)\right\|_{\infty}+\left\|Y_{2}(0, \cdot)\right\|_{\infty}\right) \mathrm{e}^{-\phi j \varepsilon} \varepsilon,
\end{aligned}
$$

and $Q(U)$ defined in (13). The constants $\kappa_{i}(1 \leq i \leq 4)$ will be specified in the sequel. Especially $\kappa_{3}=C \cdot \kappa_{2}$. Recall that $C$ is a large constant in the definition of $\mathcal{F}(U)$.

We claim $1 \leq W_{i} \leq 2$ if $\epsilon$ is small enough. By the boundness of $W_{i}$, one can verify that $L^{1}$ distance of $U^{\varepsilon}(t)$ and $V^{\varepsilon}(t)$ is equivalent to $\Phi\left(U^{\varepsilon}(t), V^{\varepsilon}(t)\right)$, i.e.

$$
\frac{1}{C_{1}}\left\|U^{\varepsilon}-V^{\varepsilon}\right\|_{L^{1}} \leq \Phi\left(U^{\varepsilon}, V^{\varepsilon}\right) \leq C_{1}\left\|U^{\varepsilon}-V^{\varepsilon}\right\|_{L^{1}}
$$

for some constant $C_{1}>1$. To understand the property of $\Phi$, let us prove the monotonicity of $W_{i}$ at first.

Lemma 3. For every $x \in \mathbb{R}, W_{i}(x, t)$ is piecewise constant and decreasing in $t$.

Proof When $t \in\left(t_{k}, t_{k+1}\right)$, all the terms w.r.t. $Y$ are unchanged. Thus $W_{i}$ is equivalent to the weight $W_{i}(x)$ defined for conservation laws in [13]. This is certainly decreasing in $t$. It suffices to verify the case $t=t_{k}$. Set

$$
W_{i}^{+} \doteq W_{i}\left(t_{k}, x\right), \quad W_{i}^{-} \doteq W_{i}\left(t_{k}-, x\right) .
$$

Using (15), we calculate that

$$
\begin{aligned}
W_{i}^{+}-W_{i}^{-} \leq & \kappa_{2} \overline{\mathcal{F}} B\left(\left\|Y_{1}(0, \cdot)\right\|_{\infty}+\left\|Y_{2}(0, \cdot)\right\|_{\infty}\right) \cdot \mathrm{e}^{-\phi k \varepsilon} \varepsilon \\
& -\kappa_{4}\left(\left\|Y_{1}(0, \cdot)\right\|_{\infty}+\left\|Y_{2}(0, \cdot)\right\|_{\infty}\right) \cdot \mathrm{e}^{-\phi k \varepsilon} \varepsilon \\
\leq & -\frac{1}{2} \kappa_{4}\left(\left\|Y_{1}(0, \cdot)\right\|_{\infty}+\left\|Y_{2}(0, \cdot)\right\|_{\infty}\right) \cdot \mathrm{e}^{-\phi k \varepsilon} \varepsilon \\
< & 0
\end{aligned}
$$

if $\kappa_{4}$ is so large that $\kappa_{2} \overline{\mathcal{F}} B<\frac{1}{4} \kappa_{4}$. The proof is completed.

The modified weight $W_{i}$ is absolutely a key factor in the whole paper. Using its monotonicity, we successfully prove in Lemma 7 that $\Phi$ is almost deceasing. This fact implies the stability and uniqueness of entropy solution. 
According to the scheme stated in Subsection 2.1 time $t$ can be categorized into three cases as follows. The time when approaching waves interact is denoted by $t^{*}$; The time when chemical reaction is activated is denoted by $t_{k}$; The other time is denoted by $t^{\circ}$. Obviously Lyapunov functional $\Phi\left(U^{\varepsilon}(t), V^{\varepsilon}(t)\right)$ is decreasing at time $t^{*}$, because the main factor

$$
\int_{-\infty}^{+\infty}\left(\left|q_{i}(t, x)\right|+\kappa_{1}\left|Y_{1}(t, x)-Y_{2}(t, x)\right|\right) d x
$$

in definition (23) is continuous at $t^{*}$, meanwhile, $W_{i}$ is decreasing steeply. Hence it suffices to discuss the remaining cases $t=t_{k}$ or $t^{\circ}$.

\subsection{Estimate of $\Phi(t)$ at $t_{k}$}

Define the components of $U^{\varepsilon}$ on two banks of discontinuity $t=t_{k}$ by

$$
\begin{aligned}
\mathcal{U}_{1}^{+} \doteq \mathcal{U}_{1}\left(t_{k}, x\right), & \mathcal{U}_{1}^{-} \doteq \mathcal{U}_{1}\left(t_{k}-, x\right) \\
Y_{1}^{+} \doteq Y_{1}\left(t_{k}, x\right), & Y_{1}^{-} \doteq Y_{1}\left(t_{k}-, x\right)
\end{aligned}
$$

And $\mathcal{U}_{2}^{ \pm}, Y_{2}^{ \pm}$represent the similar quantities of $V^{\varepsilon}$. Accordingly define $\mathbf{q}^{+}$and $\mathbf{q}^{-}$by expression (22).

Lemma 4. At every point $\left(t_{k}, x\right)$, assume $\mathbf{q}^{+}$and $\mathbf{q}^{-}$satisfy

$$
\mathcal{U}_{2}^{-}=H\left(\mathbf{q}^{-}\right)\left(\mathcal{U}_{1}^{-}\right), \quad \mathcal{U}_{2}^{+}=H\left(\mathbf{q}^{+}\right)\left(\mathcal{U}_{1}^{+}\right) .
$$

Then

$$
q_{i}^{+}-q_{i}^{-}=\mathcal{O}(1)\left\{\left|Y_{1}^{-}-Y_{2}^{-}\right| \varepsilon+\sum_{j=1}^{3}\left|q_{j}^{-}\right|\left(Y_{1}^{-}+Y_{2}^{-}\right) \varepsilon\right\} .
$$

holds for $i=1,2,3$.

The Landau symbol $\mathcal{O}(1)$ denotes a quantity whose absolute value is bounded by a constant dependent of System (11) and the domain $\mathcal{D}_{\infty}$.

Proof Set the differences

$$
\begin{aligned}
& \Delta_{1} \doteq Y_{1}^{+}-Y_{1}^{-}=-Y_{1}^{-} \phi\left(T_{1}^{-}\right) \varepsilon, \\
& \Delta_{2} \doteq Y_{2}^{+}-Y_{2}^{-}=-Y_{2}^{-} \phi\left(T_{2}^{-}\right) \varepsilon,
\end{aligned}
$$


and vector $\mathbf{w} \doteq(0,0,-q)$. Then it follows that

$$
\mathcal{U}_{1}^{+}=\mathcal{U}_{1}^{-}+\mathbf{w} \cdot \Delta_{1}, \quad \mathcal{U}_{2}^{+}=\mathcal{U}_{2}^{-}+\mathbf{w} \cdot \Delta_{2}
$$

Equations (26) and (28) determine an implicit $C^{2}$ function

$$
\mathbf{q}^{+} \doteq \mathbf{q}\left(\mathbf{q}^{-}, \Delta_{1}, \Delta_{2}\right)
$$

Notice that $\mathbf{q}(\mathbf{0}, 0,0)=\mathbf{q}\left(\mathbf{0}, \Delta_{1}, \Delta_{1}\right)=\mathbf{0}$, and $\mathbf{q}\left(\mathbf{q}^{-}, 0,0\right)=\mathbf{q}^{-}$. Using Proposition 1 in [8] and Taylor's formula, we figure out

$$
\begin{aligned}
\mathbf{q}^{+} & =\mathbf{q}\left(\mathbf{0}, \Delta_{1}, \Delta_{2}\right)+\mathbf{q}\left(\mathbf{q}^{-}, 0, \Delta_{2}\right)-\mathbf{q}\left(\mathbf{0}, 0, \Delta_{2}\right)+\mathcal{O}(1)\left\|\mathbf{q}^{-}\right\| \cdot\left|\Delta_{1}\right| \cdot \mathbf{I} \\
& =\mathbf{q}\left(\mathbf{0}, \Delta_{1}, \Delta_{2}\right)+\mathbf{q}^{-}+\mathcal{O}(1)\left\|\mathbf{q}^{-}\right\| \cdot\left(\left|\Delta_{1}\right|+\left|\Delta_{2}\right|\right) \cdot \mathbf{I} \\
& =\mathbf{q}^{-}+\mathcal{O}(1)\left|\Delta_{1}-\Delta_{2}\right| \cdot \mathbf{I}+\mathcal{O}(1)\left\|\mathbf{q}^{-}\right\| \cdot\left(\left|\Delta_{1}\right|+\left|\Delta_{2}\right|\right) \cdot \mathbf{I}
\end{aligned}
$$

where $\mathbf{I}=(1,1,1)^{T}$ is a $3 \times 1$ vector. By Lipschitz continuity of $\phi(T)$, we have

$$
\left|\phi\left(T_{1}^{-}\right)-\phi\left(T_{2}^{-}\right)\right| \leq \mathcal{O}(1)|| \mathcal{U}_{1}^{-}-\mathcal{U}_{2}^{-} \| \leq \mathcal{O}(1) \sum_{j=1}^{3}\left|q_{j}^{-}\right| .
$$

It follows from condition (14) and (30) that

$$
\begin{aligned}
\left|\Delta_{1}-\Delta_{2}\right| & \leq\left|Y_{1}^{-}-Y_{2}^{-}\right| \phi\left(T_{1}^{-}\right) \varepsilon+Y_{2}^{-}\left|\phi\left(T_{1}^{-}\right)-\phi\left(T_{2}^{-}\right)\right| \varepsilon \\
& \leq \mathcal{O}(1)\left|Y_{1}^{-}-Y_{2}^{-}\right| \varepsilon+\mathcal{O}(1) Y_{2}^{-}|| \mathcal{U}_{1}^{-}-\mathcal{U}_{2}^{-}|| \varepsilon \\
& \leq \mathcal{O}(1)\left\{\left|Y_{1}^{-}-Y_{2}^{-}\right| \varepsilon+\sum_{j=1}^{3}\left|q_{j}^{-}\right| Y_{2}^{-} \varepsilon\right\}, \\
\left|\Delta_{1}\right|+\left|\Delta_{2}\right| & \leq \mathcal{O}(1)\left(Y_{1}^{-}+Y_{2}^{-}\right) \varepsilon .
\end{aligned}
$$

Finally (29) (31) and (32) yield estimate (27).

Lemma 5. At every point $\left(t_{k}, x\right)$, components $Y$ of $U^{\varepsilon}$ and $V^{\varepsilon}$ satisfy that

$$
\left|Y_{1}^{+}-Y_{2}^{+}\right|-\left|Y_{1}^{-}-Y_{2}^{-}\right| \leq-\left|Y_{1}^{-}-Y_{2}^{-}\right| \phi \varepsilon+\mathcal{O}(1) \sum_{j=1}^{3}\left|q_{j}^{-}\right| Y_{2}^{-} \varepsilon .
$$

Proof Note that $\phi(T)$ is bounded below by $\phi$. It is easy to check that

$$
\left|Y_{1}^{+}-Y_{2}^{+}\right|=\left|Y_{1}^{-}\left(1-\phi\left(T_{1}^{-}\right) \varepsilon\right)-Y_{2}^{-}\left(1-\phi\left(T_{2}^{-}\right) \varepsilon\right)\right|
$$




$$
\begin{aligned}
& \leq\left|Y_{1}^{-}-Y_{2}^{-}\right| \cdot\left|1-\phi\left(T_{1}^{-}\right) \varepsilon\right|+Y_{2}^{-}\left|\phi\left(T_{1}^{-}\right)-\phi\left(T_{2}^{-}\right)\right| \varepsilon \\
& \leq\left|Y_{1}^{-}-Y_{2}^{-}\right| \cdot(1-\phi \varepsilon)+\mathcal{O}(1) \sum_{j=1}^{3}\left|q_{j}^{-}\right| Y_{2}^{-} \varepsilon
\end{aligned}
$$

for small $\varepsilon$. This implies the estimate (33).

Lemma 6. At every time $t_{k}=k \varepsilon$, Lyapunov functional is decreasing, i.e.

$$
\Phi\left(U^{\varepsilon}\left(t_{k}\right), V^{\varepsilon}\left(t_{k}\right)\right) \leq \Phi\left(U^{\varepsilon}\left(t_{k}-\right), V^{\varepsilon}\left(t_{k}-\right)\right) .
$$

Proof By Lemmas 40 5 and estimate (15) we have

$$
\begin{aligned}
& \left(\left|q_{i}^{+}\right|+\kappa_{1}\left|Y_{1}^{+}-Y_{2}^{+}\right|\right)-\left(\left|q_{i}^{-}\right|+\kappa_{1}\left|Y_{1}^{-}-Y_{2}^{-}\right|\right) \\
= & \left(\left|q_{i}^{+}\right|-\left|q_{i}^{-}\right|\right)+\kappa_{1}\left(\left|Y_{1}^{+}-Y_{2}^{+}\right|-\left|Y_{1}^{-}-Y_{2}^{-}\right|\right) \\
\leq & \left(-\kappa_{1} \phi+\mathcal{O}(1)\right)\left|Y_{1}^{-}-Y_{2}^{-}\right| \varepsilon+\mathcal{O}(1) \sum_{j=1}^{3}\left|q_{j}^{-}\right|\left(Y_{1}^{-}+Y_{2}^{-}\right) \varepsilon \\
\leq & -\frac{1}{2} \kappa_{1} \phi\left|Y_{1}^{-}-Y_{2}^{-}\right| \varepsilon+\mathcal{O}(1) \sum_{j=1}^{3}\left|q_{j}^{-}\right|\left(Y_{1}^{-}+Y_{2}^{-}\right) \varepsilon \\
\leq & \mathcal{O}(1) \sum_{j=1}^{3}\left|q_{j}^{-}\right| \cdot\left(\left\|Y_{1}(0, \cdot)\right\|_{\infty}+\left\|Y_{2}(0, \cdot)\right\|_{\infty}\right) \mathrm{e}^{-\phi k \varepsilon} \varepsilon
\end{aligned}
$$

provided $\kappa_{1}$ is large enough. Then estimates (34) and (25) give

$$
\begin{aligned}
& \left(\left|q_{i}^{+}\right|+\kappa_{1}\left|Y_{1}^{+}-Y_{2}^{+}\right|\right) W_{i}^{+}-\left(\left|q_{i}^{-}\right|+\kappa_{1}\left|Y_{1}^{-}-Y_{2}^{-}\right|\right) W_{i}^{-} \\
= & \left\{\left(\left|q_{i}^{+}\right|-\left|q_{i}^{-}\right|\right)+\kappa_{1}\left(\left|Y_{1}^{+}-Y_{2}^{+}\right|-\left|Y_{1}^{-}-Y_{2}^{-}\right|\right)\right\} W_{i}^{+} \\
& +\left(\left|q_{i}^{-}\right|+\kappa_{1}\left|Y_{1}^{-}-Y_{2}^{-}\right|\right)\left(W_{i}^{+}-W_{i}^{-}\right) \\
\leq & \mathcal{O}(1) \sum_{j=1}^{3}\left|q_{j}^{-}\right| \cdot\left(\left\|Y_{1}(0, \cdot)\right\|_{\infty}+\left\|Y_{2}(0, \cdot)\right\|_{\infty}\right) \mathrm{e}^{-\phi k \varepsilon} \varepsilon \cdot W_{i}^{+} \\
& -\frac{1}{2} \kappa_{4}\left(\left|q_{i}^{-}\right|+\kappa_{1}\left|Y_{1}^{-}-Y_{2}^{-}\right|\right)\left(\left\|Y_{1}(0, \cdot)\right\|_{\infty}+\left\|Y_{2}(0, \cdot)\right\|_{\infty}\right) \cdot \mathrm{e}^{-\phi k \varepsilon} \varepsilon \\
\leq & \mathcal{O}(1) \sum_{j=1}^{3}\left|q_{j}^{-}\right| \cdot\left(\left\|Y_{1}(0, \cdot)\right\|_{\infty}+\left\|Y_{2}(0, \cdot)\right\|_{\infty}\right) \mathrm{e}^{-\phi k \varepsilon} \varepsilon \\
& -\frac{1}{2} \kappa_{4}\left|q_{i}^{-}\right| \cdot\left(\left\|Y_{1}(0, \cdot)\right\|_{\infty}+\left\|Y_{2}(0, \cdot)\right\|_{\infty}\right) \cdot \mathrm{e}^{-\phi k \varepsilon} \varepsilon .
\end{aligned}
$$

Furthermore

$$
\sum_{i=1}^{3}\left(\left|q_{i}^{+}\right|+\kappa_{1}\left|Y_{1}^{+}-Y_{2}^{+}\right|\right) W_{i}^{+}-\sum_{i=1}^{3}\left(\left|q_{i}^{-}\right|+\kappa_{1}\left|Y_{1}^{-}-Y_{2}^{-}\right|\right) W_{i}^{-}
$$




$$
\begin{aligned}
\leq & \mathcal{O}(1) \sum_{j=1}^{3}\left|q_{j}^{-}\right| \cdot\left(\left\|Y_{1}(0, \cdot)\right\|_{\infty}+\left\|Y_{2}(0, \cdot)\right\|_{\infty}\right) \cdot \mathrm{e}^{-\phi k \varepsilon} \varepsilon \\
& -\frac{1}{2} \kappa_{4} \sum_{i=1}^{3}\left|q_{i}^{-}\right| \cdot\left(\left\|Y_{1}(0, \cdot)\right\|_{\infty}+\left\|Y_{2}(0, \cdot)\right\|_{\infty}\right) \cdot \mathrm{e}^{-\phi k \varepsilon} \varepsilon \\
\leq & 0
\end{aligned}
$$

provided $\kappa_{4}$ is large enough. Consequently

$$
\begin{aligned}
& \Phi\left(U^{\varepsilon}\left(t_{k}\right), V^{\varepsilon}\left(t_{k}\right)\right)-\Phi\left(U^{\varepsilon}\left(t_{k}-\right), V^{\varepsilon}\left(t_{k}-\right)\right) \\
= & \int_{-\infty}^{+\infty}\left\{\sum_{i=1}^{3}\left(\left|q_{i}^{+}\right|+\kappa_{1}\left|Y_{1}^{+}-Y_{2}^{+}\right|\right) W_{i}^{+}-\sum_{i=1}^{3}\left(\left|q_{i}^{-}\right|+\kappa_{1}\left|Y_{1}^{-}-Y_{2}^{-}\right|\right) W_{i}^{-}\right\} d x \\
\leq & 0 .
\end{aligned}
$$

\subsection{Estimate of $\Phi(t)$ at $t^{\circ}$}

At any time $t^{\circ}$, jump $\alpha \in \mathcal{J}$ locates at $x_{\alpha}$ and moves at the speed of $\dot{x}_{\alpha}$. We use the notations

$$
\begin{array}{ll}
W_{i}^{\alpha+} \doteq W_{i}\left(t^{\circ}, x_{\alpha}+\right), & W_{i}^{\alpha-} \doteq W_{i}\left(t^{\circ}, x_{\alpha}-\right), \\
q_{i}^{\alpha+} \doteq q_{i}\left(t^{\circ}, x_{\alpha}+\right), & q_{i}^{\alpha-} \doteq q_{i}\left(t^{\circ}, x_{\alpha}-\right),
\end{array}
$$

etc. to represent the quantities on two banks of $x_{\alpha}$. Then we find $\Phi$ is almost decreasing at $t^{\circ}$.

Lemma 7. At every $t=t^{\circ}$, Lyapunov functional satisfies

$$
\frac{d}{d t} \Phi\left(U^{\varepsilon}(t), V^{\varepsilon}(t)\right) \leq \mathcal{O}(1) \varepsilon
$$

Proof We can calculate the derivative of $\Phi$ by means of the technique in [13]. Set $w_{0}=\mathcal{U}_{1}, w_{i}=H_{i}\left(q_{i}\right) \circ \cdots \circ H_{1}\left(q_{1}\right)\left(\mathcal{U}_{1}\right), w_{3}=\mathcal{U}_{2}$. Then define $\lambda_{i} \doteq \lambda_{i}\left(w_{i-1}, w_{i}\right)$ as the speed of the $i$-shock connecting states $w_{i-1}$ and $w_{i}$. 
We derive that

$$
\begin{aligned}
\frac{d}{d t} \Phi\left(U^{\varepsilon}(t), V^{\varepsilon}(t)\right)= & \sum_{\alpha \in \mathcal{J}} \sum_{i=1}^{3}\left\{\left(\left|q_{i}^{\alpha-}\right|+\kappa_{1}\left|Y_{1}-Y_{2}\right|^{\alpha-}\right) W_{i}^{\alpha-}\right. \\
& \left.-\left(\left|q_{i}^{\alpha+}\right|+\kappa_{1}\left|Y_{1}-Y_{2}\right|^{\alpha+}\right) W_{i}^{\alpha+}\right\} \cdot \dot{x}_{\alpha} \\
= & \sum_{\alpha \in \mathcal{J}} \sum_{i=1}^{3}\left\{\left(\left|q_{i}^{\alpha+}\right|+\kappa_{1}\left|Y_{1}-Y_{2}\right|^{\alpha+}\right) W_{i}^{\alpha+}\left(\lambda_{i}^{\alpha+}-\dot{x}_{\alpha}\right)\right. \\
& \left.\quad-\left(\left|q_{i}^{\alpha-}\right|+\kappa_{1}\left|Y_{1}-Y_{2}\right|^{\alpha-}\right) W_{i}^{\alpha-}\left(\lambda_{i}^{\alpha-}-\dot{x}_{\alpha}\right)\right\} \\
= & \sum_{\alpha \in \mathcal{J}} \sum_{i=1}^{3} E_{i, \alpha}
\end{aligned}
$$

where

$$
\begin{aligned}
E_{i, \alpha} \doteq & \left(\left|q_{i}^{\alpha+}\right|+\kappa_{1}\left|Y_{1}-Y_{2}\right|^{\alpha+}\right) W_{i}^{\alpha+}\left(\lambda_{i}^{\alpha+}-\dot{x}_{\alpha}\right) \\
& -\left(\left|q_{i}^{\alpha-}\right|+\kappa_{1}\left|Y_{1}-Y_{2}\right|^{\alpha-}\right) W_{i}^{\alpha-}\left(\lambda_{i}^{\alpha-}-\dot{x}_{\alpha}\right) .
\end{aligned}
$$

Observe $\left|Y_{1}-Y_{2}\right|^{\alpha-}=\left|Y_{1}-Y_{2}\right|^{\alpha+}$ at $t^{\circ}$. One compute that

$$
\begin{aligned}
E_{i, \alpha}= & \left(\left|q_{i}^{\alpha+}\right|-\mid q_{i}^{\alpha-}\right) W_{i}^{\alpha+}\left(\lambda_{i}^{\alpha+}-\dot{x}_{\alpha}\right) \\
& +\left(\left|q_{i}^{\alpha-}\right|+\kappa_{1}\left|Y_{1}-Y_{2}\right|^{\alpha-}\right)\left(W_{i}^{\alpha+}-W_{i}^{\alpha-}\right)\left(\lambda_{i}^{\alpha+}-\dot{x}_{\alpha}\right) \\
& +\left(\left|q_{i}^{\alpha-}\right|+\kappa_{1}\left|Y_{1}-Y_{2}\right|^{\alpha-}\right) W_{i}^{\alpha-}\left(\lambda_{i}^{\alpha+}-\lambda_{i}^{\alpha-}\right) .
\end{aligned}
$$

Let $S, R$ and NP respectively denote the front of shock, rarefaction and nonphysical wave. Since $\left|q_{i}^{\alpha-}\right|+\kappa_{1}\left|Y_{1}-Y_{2}\right|^{\alpha-}$ is a bounded quantity, we claim that

$$
\begin{array}{ll}
\sum_{i=1}^{3} E_{i, \alpha} \leq \mathcal{O}(1) \cdot\left|\sigma_{\alpha}\right|, & \alpha \in N P \\
\sum_{i=1}^{3} E_{i, \alpha} \leq \mathcal{O}(1) \cdot \varepsilon|\alpha|, & \alpha \in S \cup R
\end{array}
$$

following from the conclusions (3.5)(3.6) in [13]. Recall that $\sum_{\alpha \in N P}\left|\sigma_{\alpha}\right| \leq \varepsilon$. Then it yields

$$
\frac{d}{d t} \Phi(U(t), V(t))=\sum_{\alpha \in N P} \sum_{i=1}^{3} E_{i, \alpha}+\sum_{\alpha \in S \cup R} \sum_{i=1}^{3} E_{i, \alpha} \leq \mathcal{O}(1) \varepsilon .
$$

In summary, we draw the following conclusion from Lemmas $6+$. 7 
Lemma 8. Let $U^{\varepsilon}(t), V^{\varepsilon}(t)$ be $\varepsilon$-approximate solutions of (1) constructed by fractional step front tracking method. Choose suitable constants $\epsilon, \kappa_{1}, \kappa_{2}, \kappa_{3}, \kappa_{4}$. If $U^{\varepsilon}(0), V^{\varepsilon}(0) \in \mathcal{D}_{0}$, then there exists a constant $C_{2}$ such that

$$
\begin{aligned}
& \Phi\left(U^{\varepsilon}(t), V^{\varepsilon}(t)\right)-\Phi\left(U^{\varepsilon}(s), V^{\varepsilon}(s)\right) \leq C_{2} \varepsilon(t-s), \\
& \left\|U^{\varepsilon}(t)-V^{\varepsilon}(t)\right\|_{L^{1}} \leq C_{1}^{2}\left\|U^{\varepsilon}(s)-V^{\varepsilon}(s)\right\|_{L^{1}}+C_{1} C_{2} \varepsilon(t-s)
\end{aligned}
$$

for all $t>s \geq 0$, where constant $C_{1}$ is specified in 24).

Remark 2. Letting $G \equiv \mathbf{0}$ or $Y_{0}=0$, one can derive the stability estimate for $\mathcal{S}_{t}^{\varepsilon}(U)$; that is,

$$
\left\|\mathcal{S}_{t}^{\varepsilon}(U)-\mathcal{S}_{t}^{\varepsilon}(V)\right\|_{L^{1}} \leq C_{1}^{2}\left\|\mathcal{S}_{s}^{\varepsilon}(U)-\mathcal{S}_{s}^{\varepsilon}(V)\right\|_{L^{1}}+C_{1} C_{2} \varepsilon(t-s)
$$

for all $t>s \geq 0$. This coincides with the result of conservation laws in [13]. Besides, Lipschitz continuity of $\mathcal{S}_{t}^{\varepsilon}(U)$ w.r.t time $t$ actually holds. Therefore we assert that there exists a Lipschitz constant $C_{3}$ such that

$$
\left\|\mathcal{S}_{t}^{\varepsilon}(U)-\mathcal{S}_{s}^{\varepsilon}(U)\right\|_{L^{1}} \leq C_{3}(t-s)
$$

for any $t>s \geq 0$. See [15, 16].

Lemma 8 and Lemma 15 on convergence give rise to the stability of entropy solution.

\section{Uniqueness of Limit Solution}

In the section we attempt to prove that by means of the scheme in Subsection 2.1. every sequence of approximate solutions must converge to a unique limit. And then give a rigorous proof of Theorem 1 .

\subsection{Estimates of Operator $\mathcal{P}_{t}^{\varepsilon}$}

Note that $\mathcal{S}_{t}^{\varepsilon}$ is identity mapping w.r.t. component $Y$. We claim the following Lemma developed from [2] still holds for non-strictly hyperbolic system (10). Its proof in detail is referred to Theorem 4.1 in [2]. 
Lemma 9. Assume that $U$ and $V$ are piecewise constant functions in $\mathcal{D}_{s} . \omega(x) \in$ $L^{1}\left(\mathbb{R}^{4}\right)$ is a piecewise constant function with small total variation, and has finitely many discontinuities. Then

$$
\left\|\mathcal{S}_{t}^{\varepsilon}(U)-\mathcal{S}_{t}^{\varepsilon}(V)-\omega\right\|_{L^{1}} \leq \mathcal{O}(1)\|U-V-\omega\|_{L^{1}}+\mathcal{O}(1) t\{\varepsilon+T . V .(\omega)\} .
$$

Additionally we need some information about source $G$. Since $G(U)$ in (1) (3) is locally Lipschitz continuous, there exists a constant $L^{\prime}>0$ such that

$$
\begin{aligned}
& \|G(U)-G(V)\| \leq L^{\prime}\|U-V\|, \\
& T . V .(G(U)) \leq L^{\prime} \cdot T . V .(U), \\
& \|G(U)\|_{L^{1}}=\left\|G(U)-G\left(U_{\infty}\right)\right\|_{L^{1}} \leq L^{\prime}\left\|U-U_{\infty}\right\|_{L^{1}}
\end{aligned}
$$

for any $U, V \in \mathcal{D}_{\infty}$. Next we can calculate the error caused by commutation of $\mathcal{T}_{t}$ and $\mathcal{S}_{t}^{\varepsilon}$.

Lemma 10. (Commutation estimate) Assume that $U$ is a piecewise constant function in $\mathcal{D}_{s}$. Then

$$
\left\|\mathcal{S}_{t}^{\varepsilon} \mathcal{T}_{t}(U)-\mathcal{T}_{t} \mathcal{S}_{t}^{\varepsilon}(U)\right\|_{L^{1}} \leq \mathcal{O}(1) t^{2}+\mathcal{O}(1) t \varepsilon
$$

holds for $\varepsilon \leq t<\bar{t}$.

Proof The Lipschitz continuity of $G$ implies

$$
T . V .\left(G\left(\mathcal{S}_{t}^{\varepsilon}(U)\right) \leq L^{\prime} \cdot T . V .\left(\mathcal{S}_{t}^{\varepsilon}(U)\right) \leq \mathcal{O}(1) \epsilon_{\infty} .\right.
$$

Combining this with Lemma 9 and Remark 2, we deduce

$$
\begin{aligned}
& \left\|\mathcal{S}_{t}^{\varepsilon} \mathcal{T}_{t}(U)-\mathcal{T}_{t} \mathcal{S}_{t}^{\varepsilon}(U)\right\|_{L^{1}} \\
= & \left\|\mathcal{S}_{t}^{\varepsilon}(U+t G(U))-\mathcal{S}_{t}^{\varepsilon}(U)-t G\left(\mathcal{S}_{t}^{\varepsilon}(U)\right)\right\|_{L^{1}} \\
\leq & \mathcal{O}(1)\left\|(U+t G(U))-U-t G\left(\mathcal{S}_{t}^{\varepsilon}(U)\right)\right\|_{L^{1}}+\mathcal{O}(1) t\left\{\varepsilon+T . V \cdot\left(t G\left(\mathcal{S}_{t}^{\varepsilon}(U)\right)\right\}\right. \\
\leq & \mathcal{O}(1) t \cdot\left\|\mathcal{S}_{t}^{\varepsilon}(U)-U\right\|_{L^{1}}+\mathcal{O}(1) t \cdot\left\{\varepsilon+t \cdot T \cdot V \cdot\left(G\left(\mathcal{S}_{t}^{\varepsilon}(U)\right)\right\}\right. \\
\leq & \mathcal{O}(1) t^{2}+\mathcal{O}(1) t \varepsilon .
\end{aligned}
$$


Remark 3. Similar property on strictly hyperbolic system was also observed in [6]. Lemma 10 is indeed the basis to establish uniqueness of solutions generated by semigroup $\mathcal{P}$. It shows that commutation of $\mathcal{S}_{\varepsilon}^{\varepsilon}$ and $\mathcal{T}_{\varepsilon}$ once gives rise to a small error of $\varepsilon^{2}$. Since composition $\mathcal{S}_{k \varepsilon}^{\varepsilon}\left(\mathcal{T}_{\varepsilon}\right)^{k}$ can be transformed to $\mathcal{P}_{k \varepsilon}^{\varepsilon}$ by $k(k-1) / 2$ commutations, the total errors accumulated in such process is less than $k^{2} \varepsilon^{2}$. The fact guarantees the conclusion in Lemma 12.

To compute the error of $\mathcal{P}_{t}^{\varepsilon}(U)-\mathcal{S}_{t}^{\varepsilon} \mathcal{T}_{t}(U)$, we establish the estimates of operator $\left(\mathcal{T}_{\varepsilon}\right)^{k}$ in advance.

Lemma 11. For any piecewise constant functions $U, V \in \mathcal{D}_{s}$, the following relations hold.

$$
\begin{aligned}
& \left\|\left(\mathcal{T}_{\varepsilon}\right)^{k}(U)-U_{\infty}\right\|_{L^{1}} \leq \mathrm{e}^{k \varepsilon L^{\prime}}\left\|U-U_{\infty}\right\|_{L^{1}}, \\
& \left\|\left(\mathcal{T}_{\varepsilon}\right)^{k}(U)-U\right\|_{L^{1}} \leq k \varepsilon L^{\prime} \mathrm{e}^{k \varepsilon L^{\prime}}\left\|U-U_{\infty}\right\|_{L^{1}}, \\
& \left\|\left(\mathcal{T}_{\varepsilon}\right)^{k}(U)-\left(\mathcal{T}_{\varepsilon}\right)^{k}(V)\right\|_{L^{1}} \leq \mathrm{e}^{k \varepsilon L^{\prime}}\|U-V\|_{L^{1}}
\end{aligned}
$$

Proof The last inequality is obvious according to Lipschitz continuity of $G$. It suffices to prove the first two inequalities by induction. Firstly, for $k=1$, one deduce from (35) that

$$
\begin{aligned}
\left\|\mathcal{T}_{\varepsilon}(U)-U_{\infty}\right\|_{L^{1}} & =\left\|U-U_{\infty}+\varepsilon\left\{G(U)-G\left(U_{\infty}\right)\right\}\right\|_{L^{1}} \\
& \leq\left\|U-U_{\infty}\right\|_{L^{1}}+\varepsilon L^{\prime}\left\|U-U_{\infty}\right\|_{L^{1}} \\
& \leq \mathrm{e}^{\varepsilon L^{\prime}}\left\|U-U_{\infty}\right\|_{L^{1}}, \\
\left\|\mathcal{T}_{\varepsilon}(U)-U\right\|_{L^{1}} & =\varepsilon\left\|G(U)-G\left(U_{\infty}\right)\right\|_{L^{1}} \\
& \leq \varepsilon L^{\prime}\left\|U-U_{\infty}\right\|_{L^{1}} \\
& \leq \varepsilon L^{\prime} \mathrm{e}^{\varepsilon L^{\prime}}\left\|U-U_{\infty}\right\|_{L^{1}} .
\end{aligned}
$$

Secondly, for integer $k>1$, we have

$$
\begin{aligned}
\left\|\left(\mathcal{T}_{\varepsilon}\right)^{k}(U)-U_{\infty}\right\|_{L^{1}} & =\left\|\left(\mathcal{T}_{\varepsilon}\right)^{k-1}(U)-U_{\infty}+\varepsilon\left\{G\left(\left(\mathcal{T}_{\varepsilon}\right)^{k-1}(U)\right)-G\left(U_{\infty}\right)\right\}\right\|_{L^{1}} \\
& \left.\leq\left(1+\varepsilon L^{\prime}\right) \| \mathcal{T}_{\varepsilon}\right)^{k-1}(U)-U_{\infty} \|_{L^{1}} \\
& \left.\leq \mathrm{e}^{\varepsilon L^{\prime}} \| \mathcal{T}_{\varepsilon}\right)^{k-1}(U)-U_{\infty} \|_{L^{1}}
\end{aligned}
$$




$$
\leq \mathrm{e}^{k \varepsilon L^{\prime}}\left\|U-U_{\infty}\right\|_{L^{1}}
$$

and

$$
\begin{aligned}
\left\|\left(\mathcal{T}_{\varepsilon}\right)^{k}(U)-U\right\|_{L^{1}} & =\left\|\left(\mathcal{T}_{\varepsilon}\right)^{k-1}(U)-U+\varepsilon\left\{G\left(\left(\mathcal{T}_{\varepsilon}\right)^{k-1}(U)\right)-G\left(U_{\infty}\right)\right\}\right\|_{L^{1}} \\
& \leq\left\|\left(\mathcal{T}_{\varepsilon}\right)^{k-1}(U)-U\right\|_{L^{1}}+\varepsilon L^{\prime}\left\|\left(\mathcal{T}_{\varepsilon}\right)^{k-1}(U)-U_{\infty}\right\|_{L^{1}} \\
& \leq(k-1) \varepsilon L^{\prime} \mathrm{e}^{(k-1) \varepsilon L^{\prime}}\left\|U-U_{\infty}\right\|_{L^{1}}+\varepsilon L^{\prime} \mathrm{e}^{(k-1) \varepsilon L^{\prime}}\left\|U-U_{\infty}\right\|_{L^{1}} \\
& \leq k \varepsilon L^{\prime} \mathrm{e}^{k \varepsilon L^{\prime}}\left\|U-U_{\infty}\right\|_{L^{1}}
\end{aligned}
$$

by induction.

Lemma 12. Assume that $U \in \mathcal{D}_{s}$ is piecewise constant. For any time $t<\bar{t}$ and positive $\varepsilon \ll \min \left\{t, t^{2}\right\}$, we have

$$
\left\|\mathcal{P}_{t}^{\varepsilon}(U)-\mathcal{S}_{t}^{\varepsilon} \mathcal{T}_{t}(U)\right\|_{L^{1}} \leq \mathcal{O}(1)\left(1+\left\|U-U_{\infty}\right\|_{L^{1}}\right) t^{2}
$$

Proof Set integer $n=[t / \varepsilon]$ and $k=1,2, \cdots, n$. Define diameters

$$
\begin{aligned}
& d_{k} \doteq \sup _{V \in \mathcal{D}_{s+k \varepsilon}}\left\|\left(\mathcal{T}_{\varepsilon}\right)^{k} \mathcal{S}_{\varepsilon}^{\varepsilon}(V)-\mathcal{S}_{\varepsilon}^{\varepsilon}\left(\mathcal{T}_{\varepsilon}\right)^{k}(V)\right\|_{L^{1}} \\
& \bar{d}_{k} \doteq \sup _{V \in \mathcal{D}_{s+k \varepsilon}}\left\|\mathcal{P}_{k \varepsilon}^{\varepsilon}(V)-\mathcal{S}_{\varepsilon}^{\varepsilon}\left(\mathcal{T}_{\varepsilon}\right)^{k}(V)\right\|_{L^{1}}
\end{aligned}
$$

Applying Lemma 11 and definition of $d_{k}$, we deduce that

$$
\begin{aligned}
\left\|\left(\mathcal{T}_{\varepsilon}\right)^{k} \mathcal{S}_{\varepsilon}^{\varepsilon}(U)-\mathcal{S}_{\varepsilon}^{\varepsilon}\left(\mathcal{T}_{\varepsilon}\right)^{k}(U)\right\|_{L^{1}} \leq & \left\|\left(\mathcal{T}_{\varepsilon}\right)^{k-1} \mathcal{T}_{\varepsilon} \mathcal{S}_{\varepsilon}^{\varepsilon}(U)-\left(\mathcal{T}_{\varepsilon}\right)^{k-1} \mathcal{S}_{\varepsilon}^{\varepsilon} \mathcal{T}_{\varepsilon}(U)\right\|_{L^{1}} \\
& +\left\|\left(\mathcal{T}_{\varepsilon}\right)^{k-1} \mathcal{S}_{\varepsilon}^{\varepsilon} \mathcal{T}_{\varepsilon}(U)-\mathcal{S}_{\varepsilon}^{\varepsilon}\left(\mathcal{T}_{\varepsilon}\right)^{k-1} \mathcal{T}_{\varepsilon}(U)\right\|_{L^{1}} \\
\leq & \mathrm{e}^{(k-1) \varepsilon L^{\prime}}\left\|\mathcal{T}_{\varepsilon} \mathcal{S}_{\varepsilon}^{\varepsilon}(U)-\mathcal{S}_{\varepsilon}^{\varepsilon} \mathcal{T}_{\varepsilon}(U)\right\|_{L^{1}} \\
& +\left\|\left(\mathcal{T}_{\varepsilon}\right)^{k-1} \mathcal{S}_{\varepsilon}^{\varepsilon} \mathcal{T}_{\varepsilon}(U)-\mathcal{S}_{\varepsilon}^{\varepsilon}\left(\mathcal{T}_{\varepsilon}\right)^{k-1} \mathcal{T}_{\varepsilon}(U)\right\|_{L^{1}} \\
\leq & \mathrm{e}^{t L^{\prime}} d_{1}+d_{k-1} .
\end{aligned}
$$

It implies $d_{k} \leq \mathrm{e}^{t L^{\prime}} d_{1}+d_{k-1}$, furthermore,

$$
d_{k} \leq \mathrm{e}^{t L^{\prime}} k d_{1}
$$

From stability result in Lemma 8 , Remark 2 and inequality (36), we derive

$$
\left\|\mathcal{P}_{k \varepsilon}^{\varepsilon}(U)-\mathcal{S}_{k \varepsilon}^{\varepsilon}\left(\mathcal{T}_{\varepsilon}\right)^{k}(U)\right\|_{L^{1}}
$$




$$
\begin{aligned}
& \leq\left\|\mathcal{P}_{(k-1) \varepsilon}^{\varepsilon} \mathcal{T}_{\varepsilon} \mathcal{S}_{\varepsilon}^{\varepsilon}(U)-\mathcal{P}_{(k-1) \varepsilon}^{\varepsilon} \mathcal{S}_{\varepsilon}^{\varepsilon} \mathcal{T}_{\varepsilon}(U)\right\|_{L^{1}} \\
& \quad+\left\|\mathcal{P}_{(k-1) \varepsilon}^{\varepsilon} \mathcal{S}_{\varepsilon}^{\varepsilon} \mathcal{T}_{\varepsilon}(U)-\mathcal{S}_{(k-1) \varepsilon}^{\varepsilon}\left(\mathcal{T}_{\varepsilon}\right)^{k-1} \mathcal{S}_{\varepsilon}^{\varepsilon} \mathcal{T}_{\varepsilon}(U)\right\|_{L^{1}} \\
& \quad+\left\|\mathcal{S}_{(k-1) \varepsilon}^{\varepsilon}\left(\mathcal{T}_{\varepsilon}\right)^{k-1} \mathcal{S}_{\varepsilon}^{\varepsilon} \mathcal{T}_{\varepsilon}(U)-\mathcal{S}_{(k-1) \varepsilon}^{\varepsilon} \mathcal{S}_{\varepsilon}^{\varepsilon}\left(\mathcal{T}_{\varepsilon}\right)^{k-1} \mathcal{T}_{\varepsilon}(U)\right\|_{L^{1}} \\
& \leq C_{1}^{2}\left\|\mathcal{T}_{\varepsilon} \mathcal{S}_{\varepsilon}^{\varepsilon}(U)-\mathcal{S}_{\varepsilon}^{\varepsilon} \mathcal{T}_{\varepsilon}(U)\right\|_{L^{1}}+C_{1} C_{2}(k-1) \varepsilon^{2} \\
& \quad+\left\|\mathcal{P}_{(k-1) \varepsilon}^{\varepsilon} \mathcal{S}_{\varepsilon}^{\varepsilon} \mathcal{T}_{\varepsilon}(U)-\mathcal{S}_{(k-1) \varepsilon}^{\varepsilon}\left(\mathcal{T}_{\varepsilon}\right)^{k-1} \mathcal{S}_{\varepsilon}^{\varepsilon} \mathcal{T}_{\varepsilon}(U)\right\|_{L^{1}} \\
& \quad+C_{1}^{2}\left\|\left(\mathcal{T}_{\varepsilon}\right)^{k-1} \mathcal{S}_{\varepsilon}^{\varepsilon} \mathcal{T}_{\varepsilon}(U)-\mathcal{S}_{\varepsilon}^{\varepsilon}\left(\mathcal{T}_{\varepsilon}\right)^{k-1} \mathcal{T}_{\varepsilon}(U)\right\|_{L^{1}} \\
& \quad+C_{1} C_{2}(k-1) \varepsilon^{2} \\
& \leq C_{1}^{2} d_{1}+\bar{d}_{k-1}+C_{1}^{2} d_{k-1}+2 C_{1} C_{2} k \varepsilon^{2} \\
& \leq C_{1}^{2}\left(1+\mathrm{e}^{t L^{\prime}} k\right) d_{1}+\bar{d}_{k-1}+2 C_{1} C_{2} k \varepsilon^{2} .
\end{aligned}
$$

Hence there holds

$$
\bar{d}_{k} \leq C_{1}^{2}\left(1+\mathrm{e}^{t L^{\prime}} k\right) d_{1}+\bar{d}_{k-1}+2 C_{1} C_{2} k \varepsilon^{2},
$$

and then

$$
\begin{aligned}
\bar{d}_{n} & \leq C_{1}^{2}\left(1+\mathrm{e}^{t L^{\prime}} n\right) n d_{1}+2 C_{1} C_{2} n^{2} \varepsilon^{2} \\
& =C_{1}^{2}\left\{n \varepsilon^{2}+\mathrm{e}^{t L^{\prime}}(n \varepsilon)^{2}\right\} \frac{d_{1}}{\varepsilon^{2}}+2 C_{1} C_{2} n^{2} \varepsilon^{2} \\
& \leq C_{1}^{2}\left(t \varepsilon+\mathrm{e}^{t L^{\prime}} t^{2}\right) \frac{d_{1}}{\varepsilon^{2}}+2 C_{1} C_{2} t^{2} \\
& \leq \mathcal{O}(1) t^{2},
\end{aligned}
$$

since $\varepsilon \leq t^{2}$ and $d_{1} / \varepsilon^{2}$ is bounded in light of Lemma 10. Observe that Lemma 11 implies

$$
\begin{aligned}
& \left\|\left(\mathcal{T}_{\varepsilon}\right)^{k}(U)-\mathcal{T}_{k \varepsilon}(U)\right\|_{L^{1}} \\
= & \left\|\mathcal{T}_{\varepsilon}\left(\mathcal{T}_{\varepsilon}\right)^{k-1}(U)-\mathcal{T}_{(k-1) \varepsilon}(U)-\varepsilon G(U)\right\|_{L^{1}} \\
= & \left\|\left(\mathcal{T}_{\varepsilon}\right)^{k-1}(U)-\mathcal{T}_{(k-1) \varepsilon}(U)+\varepsilon G\left(\left(\mathcal{T}_{\varepsilon}\right)^{k-1}(U)\right)-\varepsilon G(U)\right\|_{L^{1}} \\
\leq & \left\|\left(\mathcal{T}_{\varepsilon}\right)^{k-1}(U)-\mathcal{T}_{(k-1) \varepsilon}(U)\right\|_{L^{1}}+(k-1) \varepsilon^{2} L^{\prime 2} \mathrm{e}^{(k-1) \varepsilon L^{\prime}}\left\|U-U_{\infty}\right\|_{L^{1}} .
\end{aligned}
$$

Hence

$$
\left\|\left(\mathcal{T}_{\varepsilon}\right)^{k}(U)-\mathcal{T}_{k \varepsilon}(U)\right\|_{L^{1}} \leq k^{2} \varepsilon^{2} L^{\prime 2} \mathrm{e}^{k \varepsilon L^{\prime}}\left\|U-U_{\infty}\right\|_{L^{1}},
$$


moreover,

$$
\begin{aligned}
& \left\|\left(\mathcal{T}_{\varepsilon}\right)^{n}(U)-\mathcal{T}_{t}(U)\right\|_{L^{1}} \\
\leq & \left\|\left(\mathcal{T}_{\varepsilon}\right)^{n}(U)-\mathcal{T}_{n \varepsilon}(U)\right\|_{L^{1}}+\left\|\mathcal{T}_{t}(U)-\mathcal{T}_{n \varepsilon}(U)\right\|_{L^{1}} \\
\leq & n^{2} \varepsilon^{2} L^{\prime 2} \mathrm{e}^{n \varepsilon L^{\prime}}\left\|U-U_{\infty}\right\|_{L^{1}}+(t-n \varepsilon) L^{\prime}\left\|U-U_{\infty}\right\|_{L^{1}} \\
\leq & 2 t^{2} L^{\prime 2} \mathrm{e}^{t L^{\prime}}\left\|U-U_{\infty}\right\|_{L^{1}}
\end{aligned}
$$

provided $L^{\prime}>1$. Consequently, we see that

$$
\begin{aligned}
&\left\|\mathcal{P}_{t}^{\varepsilon}(U)-\mathcal{S}_{t}^{\varepsilon} \mathcal{T}_{t}(U)\right\|_{L^{1}} \leq\left\|\mathcal{P}_{t}^{\varepsilon}(U)-\mathcal{P}_{n \varepsilon}^{\varepsilon}(U)\right\|_{L^{1}}+\left\|\mathcal{P}_{n \varepsilon}^{\varepsilon}(U)-\mathcal{S}_{n \varepsilon}^{\varepsilon} \mathcal{T}_{n \varepsilon}(U)\right\|_{L^{1}} \\
& \quad+\left\|\mathcal{S}_{n \varepsilon}^{\varepsilon} \mathcal{T}_{n \varepsilon}(U)-\mathcal{S}_{t}^{\varepsilon} \mathcal{T}_{t}(U)\right\|_{L^{1}} \\
& \leq \quad \mathcal{O}(1) \varepsilon^{2}+\bar{d}_{n}+\mathcal{O}(1)\left\|\mathcal{T}_{n \varepsilon}(U)-\mathcal{T}_{t}(U)\right\|_{L^{1}} \\
& \quad+\mathcal{O}(1)(t-n \varepsilon) \varepsilon \\
& \leq \mathcal{O}(1) \varepsilon^{2}+\mathcal{O}(1)\left(1+\left\|U-U_{\infty}\right\|_{L^{1}}\right) t^{2} \\
& \leq \mathcal{O}(1)\left(1+\left\|U-U_{\infty}\right\|_{L^{1}}\right) t^{2}
\end{aligned}
$$

by Lemma 8 . Remark 2 and estimates (37) (38).

In the end we state an approximate semigroup property of $\mathcal{P}_{t}^{\varepsilon}$.

Lemma 13. (Semigroup estimate) For any $t_{1}, t_{2}>0$ and $U \in \mathcal{D}_{s}$, the inequality

$$
\left\|\mathcal{P}_{t_{2}}^{\varepsilon} \mathcal{P}_{t_{1}}^{\varepsilon}(U)-\mathcal{P}_{t_{1}+t_{2}}^{\varepsilon}(U)\right\|_{L^{1}} \leq \mathcal{O}(1)\left(1+\varepsilon+t_{1}+t_{2}+\left\|U-U_{\infty}\right\|_{L^{1}}\right) \varepsilon
$$

holds if $0<\varepsilon \ll \bar{t}$.

Proof Fix $\varepsilon \ll \bar{t}$. Then there exists two integers $k$ and $K$ such that

$$
(k-1) \varepsilon<t_{1} \leq k \varepsilon, \quad K \varepsilon \leq t_{1}+t_{2}<(K+1) \varepsilon
$$

They yield

$$
\left[\frac{t_{2}}{\varepsilon}\right]=K-k \text { or } \quad K-k+1 .
$$

Since the arguments of these two cases are analogous, we only discuss the case $\left[t_{2} / \varepsilon\right]=K-k$. 
Let $V \doteq \mathcal{P}_{t_{1}}^{\varepsilon}(U), V_{\infty}:=\mathcal{P}_{t_{1}}^{\varepsilon}\left(U_{\infty}\right)$. Then Lemma 8 and Remark 2 give that

$$
\begin{aligned}
& \quad\left\|\mathcal{P}_{t_{2}}^{\varepsilon} \mathcal{P}_{t_{1}}^{\varepsilon}(U)-\mathcal{P}_{t_{1}+t_{2}}^{\varepsilon}(U)\right\|_{L^{1}} \\
& \leq\left\|\mathcal{S}_{t_{1}+t_{2}-K \varepsilon}^{\varepsilon} \mathcal{S}_{k \varepsilon-t_{1}}^{\varepsilon} \mathcal{P}_{(K-k) \varepsilon}^{\varepsilon}(V)-\mathcal{S}_{t_{1}+t_{2}-K \varepsilon}^{\varepsilon} \mathcal{P}_{(K-k) \varepsilon}^{\varepsilon} \mathcal{T}_{\varepsilon} \mathcal{S}_{k \varepsilon-t_{1}}^{\varepsilon}(V)\right\|_{L^{1}} \\
& \leq \\
& \quad+\mathcal{O}(1)\left\|\mathcal{S}_{k \varepsilon-t_{1}}^{\varepsilon} \mathcal{P}_{(K-k) \varepsilon}^{\varepsilon}(V)-\mathcal{P}_{(K-k) \varepsilon}^{\varepsilon} \mathcal{T}_{\varepsilon} \mathcal{S}_{k \varepsilon-t_{1}}^{\varepsilon}(V)\right\|_{L^{1}} \\
& \quad+\mathcal{O}(1)\left(t_{1}+t_{2}-K \varepsilon\right) \varepsilon \\
& \leq \mathcal{O}(1)\left\|\mathcal{P}_{(K-k) \varepsilon}^{\varepsilon} \mathcal{T}_{\varepsilon} \mathcal{S}_{k \varepsilon-t_{1}}^{\varepsilon}(V)-\mathcal{P}_{(K-k) \varepsilon}^{\varepsilon}(V)\right\|_{L^{1}} \\
& \quad+\mathcal{O}(1)\left\|\mathcal{S}_{k \varepsilon-t_{1}}^{\varepsilon} \mathcal{P}_{(K-k) \varepsilon}^{\varepsilon}(V)-\mathcal{P}_{(K-k) \varepsilon}^{\varepsilon}(V)\right\|_{L^{1}}+\mathcal{O}(1) \varepsilon^{2} \\
& \leq \mathcal{O}(1)\left\|\mathcal{T}_{\varepsilon} \mathcal{S}_{k \varepsilon-t_{1}}^{\varepsilon}(V)-V\right\|_{L^{1}}+\mathcal{O}(1)(K-k) \varepsilon^{2} \\
& \quad+\mathcal{O}(1)\left(k \varepsilon-t_{1}\right)+\mathcal{O}(1) \varepsilon^{2} \\
& \leq \mathcal{O}(1)\left\{\left\|\mathcal{S}_{k \varepsilon-t_{1}}^{\varepsilon}(V)-V\right\|_{L^{1}}+\varepsilon\left\|G\left(\mathcal{S}_{k \varepsilon-t_{1}}^{\varepsilon}(V)\right)-G\left(\mathcal{S}_{k \varepsilon-t_{1}}^{\varepsilon}\left(V_{\infty}\right)\right)\right\|_{L^{1}}\right\} \\
& \quad+\mathcal{O}(1)\left(t_{2} \varepsilon+\varepsilon+\varepsilon^{2}\right) \\
& \leq \mathcal{O}(1)\left\|V-V_{\infty}\right\|_{L^{1} \varepsilon}+\mathcal{O}(1)\left(k \varepsilon-t_{1}\right) \varepsilon+\mathcal{O}(1)\left(t_{2} \varepsilon+\varepsilon+\varepsilon^{2}\right) \\
& \leq \mathcal{O}(1)\left\|U-U_{\infty}\right\|_{L^{1} \varepsilon} \varepsilon \mathcal{O}(1)\left(t_{1}+t_{2}+1+\varepsilon\right) \varepsilon .
\end{aligned}
$$

We complete the proof.

\subsection{Convergence of $\varepsilon$-approximate Solutions}

First, we need a local estimate of different trajectories $\mathcal{P}_{t}^{\mu}$ and $\mathcal{P}_{t}^{\nu}$ which originate from the same state $U$.

Lemma 14. Assume that $U \in \mathcal{D}_{s}$ is piecewise constant. Then the inequality

$$
\left\|\mathcal{P}_{t}^{\mu}(U)-\mathcal{P}_{t}^{\nu}(U)\right\|_{L^{1}} \leq \mathcal{O}(1)\left(1+\left\|U-U_{\infty}\right\|_{L^{1}}\right) t^{2}
$$

holds for any positive $\mu, \nu \ll \min \left\{t, t^{2}\right\}$, and time $t<\bar{t}$.

Proof Note that for any $V \in \mathcal{D}_{\infty}$,

$$
\left\|\mathcal{S}_{t}^{\mu}(V)-\mathcal{S}_{t}^{\nu}(V)\right\|_{L^{1}} \leq \mathcal{O}(1) \max \{\mu, \nu\} t
$$

holds (cf. [15]) . By Lemma 12 we deduce

$$
\left\|\mathcal{P}_{t}^{\mu}(U)-\mathcal{P}_{t}^{\nu}(U)\right\|_{L^{1}}
$$




$$
\begin{aligned}
& \leq\left\|\mathcal{P}_{t}^{\mu}(U)-\mathcal{S}_{t}^{\mu} \mathcal{T}_{t}(U)\right\|_{L^{1}}+\left\|\mathcal{P}_{t}^{\nu}(U)-\mathcal{S}_{t}^{\nu} \mathcal{T}_{t}(U)\right\|_{L^{1}}+\left\|\mathcal{S}_{t}^{\mu} \mathcal{T}_{t}(U)-\mathcal{S}_{t}^{\nu} \mathcal{T}_{t}(U)\right\|_{L^{1}} \\
& \leq \mathcal{O}(1)\left(1+\left\|U-U_{\infty}\right\|_{L^{1}}\right) t^{2}+\mathcal{O}(1) \max \{\mu, \nu\} t \\
& \leq \mathcal{O}(1)\left(1+\left\|U-U_{\infty}\right\|_{L^{1}}\right) t^{2}
\end{aligned}
$$

Second, we can generalize the result in Lemma 14 to that of trajectories with distinct origins. The following Lemma exhibits that $\left\{\mathcal{P}_{t}^{\varepsilon}\left(U^{\varepsilon}\right)\right\}$ is a Cauchy sequence w.r.t. $\varepsilon$ in $L^{1}$-topology.

Lemma 15. Assume that $U_{0}^{\mu}, U_{0}^{\nu}$ are piecewise constant functions in $\mathcal{D}_{0}$ such that

$$
\begin{gathered}
\left\|U_{0}^{\mu}-U_{0}\right\|_{L^{1}}<\mu, \quad\left\|U_{0}^{\nu}-U_{0}\right\|_{L^{1}}<\nu, \\
T . V .\left(U_{0}^{\mu}\right) \leq T . V .\left(U_{0}\right), \quad \text { T.V. }\left(U_{0}^{\nu}\right) \leq T . V .\left(U_{0}\right)
\end{gathered}
$$

with some $U_{0} \in \mathcal{D}_{0}$. Then for every $t \geq 0$,

$$
\left\|\mathcal{P}_{t}^{\mu}\left(U_{0}^{\mu}\right)-\mathcal{P}_{t}^{\nu}\left(U_{0}^{\nu}\right)\right\|_{L^{1}} \rightarrow 0
$$

as $\mu, \nu \rightarrow 0$.

Proof Divide the interval $[0, t]$ equally into $m$ parts such that $\Delta t=t / m$ is small. Let the points of such division satisfy

$$
0=t_{0}<t_{1}<\cdots<t_{m}=t
$$

If $\mu, \nu<\min \left\{\bar{t},(\Delta t)^{2}\right\}$, it follows from Lemma 13 that

$$
\begin{aligned}
& \quad\left\|\mathcal{P}_{t}^{\mu}\left(U_{0}^{\mu}\right)-\mathcal{P}_{t}^{\nu}\left(U_{0}^{\mu}\right)\right\|_{L^{1}} \\
& \leq \sum_{i=1}^{m}\left\|\mathcal{P}_{t-t_{i}}^{\mu} \mathcal{P}_{t_{i}}^{\nu}\left(U_{0}^{\mu}\right)-\mathcal{P}_{t-t_{i-1}}^{\mu} \mathcal{P}_{t_{i-1}}^{\nu}\left(U_{0}^{\mu}\right)\right\|_{L^{1}} \\
& \leq \sum_{i=1}^{m}\left\|\mathcal{P}_{t-t_{i}}^{\mu} \mathcal{P}_{t_{i}}^{\nu}\left(U_{0}^{\mu}\right)-\mathcal{P}_{t-t_{i}}^{\mu} \mathcal{P}_{t_{i}-t_{i-1}}^{\mu} \mathcal{P}_{t_{i-1}}^{\nu}\left(U_{0}^{\mu}\right)\right\|_{L^{1}} \\
& \quad+\mathcal{O}(1) \sum_{i=1}^{m}\left(1+\mu+t-t_{i-1}+\left\|\mathcal{P}_{t_{i-1}}^{\nu}\left(U_{0}^{\mu}\right)-U_{\infty}\right\|_{L^{1}}\right) \mu \\
& \quad: \Sigma_{1}+\Sigma_{2}
\end{aligned}
$$


where $\Sigma_{1}$ (resp. $\left.\Sigma_{2}\right)$ denotes the first (resp. the second) sum of the third part in estimate (40). Thus using Lemmas 13/14/8 and Remark 2, we obtain

$$
\begin{aligned}
\Sigma_{1} \leq & \mathcal{O}(1) \sum_{i=1}^{m}\left\|\mathcal{P}_{t_{i}}^{\nu}\left(U_{0}^{\mu}\right)-\mathcal{P}_{t_{i}-t_{i-1}}^{\mu} \mathcal{P}_{t_{i-1}}^{\nu}\left(U_{0}^{\mu}\right)\right\|_{L^{1}}+\mathcal{O}(1) \sum_{i=1}^{m}\left(t-t_{i}\right) \mu \\
\leq & \mathcal{O}(1) \sum_{i=1}^{m}\left\|\mathcal{P}_{t_{i}-t_{i-1}}^{\nu} \mathcal{P}_{t_{i-1}}^{\nu}\left(U_{0}^{\mu}\right)-\mathcal{P}_{t_{i}-t_{i-1}}^{\mu} \mathcal{P}_{t_{i-1}}^{\nu}\left(U_{0}^{\mu}\right)\right\|_{L^{1}} \\
& +\mathcal{O}(1) \sum_{i=1}^{m}\left(1+\nu+t_{i}+\left\|U_{0}^{\mu}-U_{\infty}\right\|_{L^{1}}\right) \nu+\mathcal{O}(1) \sum_{i=1}^{m}\left(t-t_{i}\right) \mu \\
\leq & \mathcal{O}(1) \sum_{i=1}^{m}\left(1+\left\|\mathcal{P}_{t_{i-1}}^{\nu}\left(U_{0}^{\mu}\right)-U_{\infty}\right\|_{L^{1}}\right)\left(t_{i}-t_{i-1}\right)^{2} \\
& +\mathcal{O}(1)\left(1+\nu+t+\left\|U_{0}^{\mu}-U_{\infty}\right\|_{L^{1}}\right) m \nu+\mathcal{O}(1) t m \mu \\
\leq & \mathcal{O}(1) \sum_{i=1}^{m}\left(1+\left\|\mathcal{P}_{t_{i-1}}^{\nu}\left(U_{0}^{\mu}\right)-\mathcal{P}_{t_{i-1}}^{\nu}\left(U_{\infty}\right)\right\|_{L^{1}}+\left\|\mathcal{S}_{t_{i-1}}^{\nu}\left(U_{\infty}\right)-U_{\infty}\right\|_{L^{1}}\right)(\Delta t)^{2} \\
& +\mathcal{O}(1)\left(1+\nu+t+\left\|U_{0}^{\mu}-U_{\infty}\right\|_{L^{1}}\right) m \nu+\mathcal{O}(1) t m \mu \\
\leq & \mathcal{O}(1) \sum_{i=1}^{m}\left(1+\nu t_{i-1}+t_{i-1}+\left\|U_{0}^{\mu}-U_{\infty}\right\|_{L^{1}}\right)(\Delta t)^{2} \\
& +\mathcal{O}(1)\left(1+\nu+t+\left\|U_{0}^{\mu}-U_{\infty}\right\|_{L^{1}}\right) m \nu+\mathcal{O}(1) t m \mu \\
\leq & \mathcal{O}(1)\left(1+\mu+\nu t+t+\left\|U_{0}-U_{\infty}\right\|_{L^{1}}\right) t \Delta t \\
& +\mathcal{O}(1)\left(1+\mu+\nu+t+\left\|U_{0}-U_{\infty}\right\|_{L^{1}}\right) m \nu+\mathcal{O}(1) t m \mu \\
& \left.\leq \mathcal{O},\left\|\mathcal{S}_{t_{i-1}}^{\nu}\left(U_{\infty}\right)-U_{\infty}\right\|_{L^{1}}\right) \mu \\
& \leq \mathcal{O}(1)\left(1+\mu+t+\nu t+\left\|U_{0}-U_{\infty}\right\|_{L^{1}}\right) m \mu \\
& \leq \mathcal{O}(1) \sum_{i=1}^{m}\left(1+\mu+t-t_{i-1}+t_{i-1}+\nu t_{i-1}+\left\|U_{0}^{\mu}-U_{\infty}\right\|_{L^{1}}\right) \mu \\
& \\
&
\end{aligned}
$$

Estimates (40)-(42) yield

$$
\left\|\mathcal{P}_{t}^{\mu}\left(U_{0}^{\mu}\right)-\mathcal{P}_{t}^{\nu}\left(U_{0}^{\mu}\right)\right\|_{L^{1}}
$$




$$
\leq \mathcal{O}(1)\left(1+\mu+\nu+t+\nu t+\left\|U_{0}-U_{\infty}\right\|_{L^{1}}\right)\{t \Delta t+m(\mu+\nu)\} .
$$

As a result,

$$
\begin{aligned}
& \left\|\mathcal{P}_{t}^{\mu}\left(U_{0}^{\mu}\right)-\mathcal{P}_{t}^{\nu}\left(U_{0}^{\nu}\right)\right\|_{L^{1}} \\
\leq & \left\|\mathcal{P}_{t}^{\mu}\left(U_{0}^{\mu}\right)-\mathcal{P}_{t}^{\nu}\left(U_{0}^{\mu}\right)\right\|_{L^{1}}+\left\|\mathcal{P}_{t}^{\nu}\left(U_{0}^{\mu}\right)-\mathcal{P}_{t}^{\nu}\left(U_{0}^{\nu}\right)\right\|_{L^{1}} \\
\leq & \mathcal{O}(1)\left(1+\mu+\nu+t+\nu t+\left\|U_{0}-U_{\infty}\right\|_{L^{1}}\right)\{t \Delta t+m(\mu+\nu)\} \\
& \quad+\mathcal{O}(1)\left\|U_{0}^{\mu}-U_{0}^{\nu}\right\|_{L^{1}}+\mathcal{O}(1) t \nu \\
\leq & \mathcal{O}(1)\left(1+\mu+\nu+t+\nu t+\left\|U_{0}-U_{\infty}\right\|_{L^{1}}\right)\{t \Delta t+m(\mu+\nu)\} .
\end{aligned}
$$

Passing $\mu, \nu \rightarrow 0$, we have

$$
\varlimsup_{\mu, \nu \rightarrow 0}\left\|\mathcal{P}_{t}^{\mu}\left(U_{0}^{\mu}\right)-\mathcal{P}_{t}^{\nu}\left(U_{0}^{\nu}\right)\right\|_{L^{1}} \leq \mathcal{O}(1)\left(1+t+\left\|U_{0}-U_{\infty}\right\|_{L^{1}}\right) t \Delta t,
$$

which implies (39) due to the arbitrariness of $\Delta t$.

In the light of previous preparation, we continue to prove Theorem 1.

\section{Proof of Theorem 1}

Assume that $\epsilon$ is small enough, and $U_{0}(x) \in \mathcal{D}_{0}$. Then according to Lemma 15. every sequence of $\varepsilon$-approximate solution $\mathcal{P}_{t}^{\varepsilon}\left(U_{0}^{\varepsilon}\right)$ to Cauchy problem (1) (2) converges to a unique limit solution $U(t, x) \doteq \mathcal{P}_{t}\left(U_{0}\right)$. It was proved in [8] $U(t, x)$ is an entropy solution to problem (11) (2). Thus it suffices to verify that the mapping $\mathcal{P}:[0,+\infty) \times \mathcal{D}_{\infty} \rightarrow \mathcal{D}_{\infty}$ is a Lipschitz semigroup.

Semigroup property (5) is deduced from uniqueness and Lemmas 13 and 15 Now we only check the Lipschitz continuity of $\mathcal{P}$. At every time $t_{k}=k \varepsilon$, one see that

$$
\begin{aligned}
\left\|\mathcal{P}_{t_{k}}^{\varepsilon}\left(U_{0}^{\varepsilon}\right)-\mathcal{P}_{t_{k}-}^{\varepsilon}\left(U_{0}^{\varepsilon}\right)\right\|_{L^{1}} & =\mathcal{O}(1) \int_{-\infty}^{+\infty} Y\left(t_{k}-, x\right) \phi\left(T\left(t_{k}-, x\right)\right) \varepsilon d x \\
& \leq \mathcal{O}(1) \mathrm{e}^{-\phi k \varepsilon} \varepsilon \int_{-\infty}^{+\infty} Y(0, x) d x
\end{aligned}
$$

according to definition (11) and decay rate in (16). By finite propagation speed of waves, we assert that

$$
\left\|\mathcal{P}_{t^{\prime}}^{\varepsilon}\left(U_{0}^{\varepsilon}\right)-\mathcal{P}_{s^{\prime}}^{\varepsilon}\left(U_{0}^{\varepsilon}\right)\right\|_{L^{1}} \leq \mathcal{O}(1) T . V .\left(\mathcal{P}_{t_{k-1}}^{\varepsilon}\left(U_{0}^{\varepsilon}\right)\right) \cdot\left|t^{\prime}-s^{\prime}\right|
$$


for any $t^{\prime}, s^{\prime} \in\left[t_{k-1}, t_{k}\right)$. Suppose $t>s \geq 0$. Choose $\varepsilon$ small enough, then have

$$
\begin{aligned}
\left\|\mathcal{P}_{t}^{\varepsilon}\left(U_{0}^{\varepsilon}\right)-\mathcal{P}_{s}^{\varepsilon}\left(U_{0}^{\varepsilon}\right)\right\|_{L^{1}} & \leq \mathcal{O}(1)\left(t-s+\sum_{s \leq k \varepsilon \leq t} \mathrm{e}^{-\phi k \varepsilon} \varepsilon\right) \\
& \leq \mathcal{O}(1)\left(t-s+\int_{s}^{t} \mathrm{e}^{-\phi \tau} d \tau\right) \\
& =\mathcal{O}(1)|t-s| .
\end{aligned}
$$

As $\varepsilon \rightarrow 0$, there holds

$$
\left\|\mathcal{P}_{t}\left(U_{0}\right)-\mathcal{P}_{s}\left(U_{0}\right)\right\|_{L^{1}} \leq \mathcal{O}(1)|t-s|
$$

Remember that Lemma 8 implies for any $U_{0}, V_{0} \in \mathcal{D}_{0}$,

$$
\left\|\mathcal{P}_{s}\left(U_{0}\right)-\mathcal{P}_{s}\left(V_{0}\right)\right\|_{L^{1}} \leq \mathcal{O}(1)\left\|U_{0}-V_{0}\right\|_{L^{1}}
$$

if passing to limits. Finally preceding two inequalities yield ([6).

\section{Local Characteristics of Entropy Weak Solutions}

This section is concerned with the local geometric features of entropy solutions. $U_{0}^{\varepsilon}$ is as usual regarded as the $\varepsilon$-approximation of initial data $U_{0} \in \mathcal{D}_{0}$. Based on Theorem 1 in this paper and the convergence of $\mathcal{S}_{t}^{\varepsilon}$ in [15], we claim that in $L^{1}$-topology sense,

$$
\begin{aligned}
U_{0}^{\varepsilon} & \rightarrow U_{0}, \\
\mathcal{P}_{t}^{\varepsilon}\left(U_{0}^{\varepsilon}\right) & \rightarrow \mathcal{P}_{t}\left(U_{0}\right), \\
\mathcal{S}_{t}^{\varepsilon} \mathcal{T}_{t}\left(U_{0}^{\varepsilon}\right) & \rightarrow \mathcal{S}_{t} \mathcal{T}_{t}\left(U_{0}\right), \\
\mathcal{T}_{t} \mathcal{S}_{t}^{\varepsilon}\left(U_{0}^{\varepsilon}\right) & \rightarrow \mathcal{T}_{t} \mathcal{S}_{t}\left(U_{0}\right),
\end{aligned}
$$

as $\varepsilon \rightarrow 0$. Hence by Lemmas 10 and 12, we draw the following conclusion.

Lemma 16. Suppose $U(s, x)=\mathcal{P}_{s}\left(U_{0}\right)$ with $U_{0} \in \mathcal{D}_{0}$. Then

$$
\begin{aligned}
& \left\|\mathcal{P}_{\theta}(U)-\mathcal{S}_{\theta} \mathcal{T}_{\theta}(U)\right\|_{L^{1}} \leq \mathcal{O}(1)\left(1+\left\|U-U_{\infty}\right\|_{L^{1}}\right) \theta^{2}, \\
& \left\|\mathcal{P}_{\theta}(U)-\mathcal{T}_{\theta} \mathcal{S}_{\theta}(U)\right\|_{L^{1}} \leq \mathcal{O}(1)\left(1+\left\|U-U_{\infty}\right\|_{L^{1}}\right) \theta^{2},
\end{aligned}
$$




$$
\left\|\mathcal{S}_{\theta} \mathcal{T}_{\theta}(U)-\mathcal{T}_{\theta} \mathcal{S}_{\theta}(U)\right\|_{L^{1}} \leq \mathcal{O}(1) \theta^{2}
$$

hold for $\theta \in(0, \bar{t})$.

We will utilize this Lemma to analyze the relations between balance laws (1) and conservation laws (resp. transport system). Now let us focus on a small neighborhood of point $(s, \xi)$. Introduce Riemann problem of conservation laws,

$$
\begin{aligned}
& w_{t}+F(w)_{x}=0, \\
& w(0, x)= \begin{cases}U(s, \xi+) & \text { if } x<\xi, \\
U(s, \xi-) & \text { if } x>\xi\end{cases}
\end{aligned}
$$

and Cauchy problem of transport equations.

$$
\begin{aligned}
& w_{t}+d F(U(s, \xi)) w_{x}=G(U(s, x)), \\
& w(0, x)=U(s, x) .
\end{aligned}
$$

Then regard $U_{\xi}^{C}$ as the entropy solution to (43), and $U_{\xi}^{T}$ as the solution to (44). One can figure out that

$$
\begin{aligned}
& U_{\xi}^{T}(t, x)=U_{\xi}^{1}+U_{\xi}^{2}, \\
& U_{\xi}^{1}(t, x)=\sum_{i=1}^{3}\left(l_{i}^{\xi} \cdot U\left(s, x-\lambda_{i}^{\xi} t\right)\right) r_{i}^{\xi}, \\
& U_{\xi}^{2}(t, x)=\sum_{i=1}^{3} \int_{0}^{t}\left(l_{i}^{\xi} \cdot G\left(U\left(s, x-\lambda_{i}^{\xi} \tau\right)\right) r_{i}^{\xi} d \tau,\right.
\end{aligned}
$$

where $l_{i}^{\xi}$ (resp. $r_{i}^{\xi}$ ) denotes the left (resp. right) eigenvector of $d F(U(s, \xi))$. Now we proceed to prove Theorem 2.

\section{Proof of Theorem 2}

Suppose $U(t, x)=\mathcal{P}_{t}\left(U_{0}\right)$ with $U_{0} \in \mathcal{D}_{0}$. It is an entropy solution to (1) (2).

Fix $s \geq 0$ and $\xi \in \mathbb{R}$. Then we compute the limit (7). Observe that

$$
\begin{aligned}
& \frac{1}{\theta} \int_{\xi-\theta \hat{\lambda}}^{\xi+\theta \hat{\lambda}}\left\|U(s+\theta, x)-U_{\xi}^{C}(\theta, x)\right\| d x \\
\leq & \frac{1}{\theta} \int_{\xi-\theta \hat{\lambda}}^{\xi+\theta \hat{\lambda}}\left\|\mathcal{P}_{\theta}(U(s, x))-S_{\theta}(U(s, x))-\theta G(U(s, x))\right\| d x
\end{aligned}
$$




$$
\begin{aligned}
& +\frac{1}{\theta} \int_{\xi-\theta \hat{\lambda}}^{\xi+\theta \hat{\lambda}}\left\|S_{\theta}(U(s, x))-U_{\xi}^{C}(\theta, x)\right\| d x+\frac{1}{\theta} \int_{\xi-\theta \hat{\lambda}}^{\xi+\theta \hat{\lambda}}\|\theta G(U(s, x))\| d x \\
\leq & \frac{1}{\theta}\left\|\mathcal{P}_{\theta}(U(s, x))-S_{\theta}(U(s, x))-\theta G(U(s, x))\right\|_{L^{1}} \\
& +\frac{1}{\theta} \int_{\xi-\theta \hat{\lambda}}^{\xi+\theta \hat{\lambda}}\left\|S_{\theta}(U(s, x))-U_{\xi}^{C}(\theta, x)\right\| d x+\int_{\xi-\theta \hat{\lambda}}^{\xi+\theta \hat{\lambda}}\|G(U(s, x))\| d x .
\end{aligned}
$$

Since

$$
\left\|\mathcal{P}_{\theta}(U)-\mathcal{T}_{t} \mathcal{S}_{\theta}(U)\right\|_{L^{1}} \leq \mathcal{O}(1)\left(1+\left\|U-U_{\infty}\right\|_{L^{1}}\right) \theta^{2}
$$

for $\theta \in(0, \bar{t})$, we have

$$
\begin{aligned}
& \lim _{\theta \rightarrow 0^{+}} \frac{1}{\theta}\left\|\mathcal{P}_{\theta}(U(s, x))-S_{\theta}(U(s, x))-\theta G(U(s, x))\right\|_{L^{1}} \\
= & \lim _{\theta \rightarrow 0^{+}} \frac{1}{\theta}\left\|\mathcal{P}_{\theta}(U(s, x))-\mathcal{T}_{\theta} \mathcal{S}_{\theta}(U(s, x))\right\|_{L^{1}} \\
= & 0
\end{aligned}
$$

Additionally

$$
\lim _{\theta \rightarrow 0^{+}} \frac{1}{\theta} \int_{\xi-\theta \hat{\lambda}}^{\xi+\theta \hat{\lambda}}\left\|S_{\theta}(U(s, x))-U_{\xi}^{C}(\theta, x)\right\| d x=0
$$

is proved by Bressan [15] (see also [16]);

$$
\lim _{\theta \rightarrow 0^{+}} \int_{\xi-\theta \hat{\lambda}}^{\xi+\theta \hat{\lambda}}\|G(U(s, x))\| d x=0
$$

is deduced from absolute continuity of Lebesgue integral. Therefore conclusion (77) holds.

Next we calculate the limit (8). For every $a<\xi<b$,

$$
\begin{aligned}
& \frac{1}{\theta} \int_{a+\theta \hat{\lambda}}^{b-\theta \hat{\lambda}}\left\|U(s+\theta, x)-U_{\xi}^{T}(\theta, x)\right\| d x \\
\leq & \frac{1}{\theta} \int_{a+\theta \hat{\lambda}}^{b-\theta \hat{\lambda}}\left\|\mathcal{P}_{\theta}(U(s))(x)-\mathcal{S}_{\theta}(U(s, x))-\theta G(U(s, x))\right\| d x \\
& +\frac{1}{\theta} \int_{a+\theta \hat{\lambda}}^{b-\theta \hat{\lambda}}\left\|\mathcal{S}_{\theta}(U(s, x))-U_{\xi}^{1}(\theta, x)\right\| d x \\
& +\frac{1}{\theta} \int_{a+\theta \hat{\lambda}}^{b-\theta \hat{\lambda}}\left\|\theta G(U(s, x))-U_{\xi}^{2}(\theta, x)\right\| d x
\end{aligned}
$$




$$
\begin{aligned}
\leq & \frac{1}{\theta}\left\|\mathcal{P}_{\theta}(U(s))(x)-\mathcal{T}_{\theta} \mathcal{S}_{\theta}(U(s, x))\right\|_{L^{1}}+\mathcal{O}(1)\{T . V .(U(s) ;(a, b))\}^{2} \\
& +\frac{1}{\theta} \int_{a+\theta \hat{\lambda}}^{b-\theta \hat{\lambda}}\left\|\theta G(U(s, x))-U_{\xi}^{2}(\theta, x)\right\| d x .
\end{aligned}
$$

The first term of the sum above tends to 0 as $\theta \rightarrow 0^{+}$. The second term was derived by [2, 15] etc.. So it suffices to compute the third term . In fact

$$
\begin{aligned}
& \frac{1}{\theta} \int_{a+\theta \hat{\lambda}}^{b-\theta \hat{\lambda}}\left\|\theta G(U(s, x))-U_{\xi}^{2}(\theta, x)\right\| d x \\
\leq & \frac{1}{\theta} \int_{a+\theta \hat{\lambda}}^{b-\theta \hat{\lambda}}\left\|\int_{0}^{\theta} \sum_{i=1}^{3} l_{i}^{\xi} \cdot G(U(s, x)) r_{i}^{\xi} d \tau-\int_{0}^{\theta} \sum_{i=1}^{3} l_{i}^{\xi} \cdot G\left(U\left(s, x-\lambda_{i}^{\xi} \tau\right)\right) r_{i}^{\xi} d \tau\right\| d x \\
\leq & \mathcal{O}(1) \frac{1}{\theta} \sum_{i=1}^{3} \int_{0}^{\theta} \int_{a+\theta \hat{\lambda}}^{b-\theta \hat{\lambda}}\left\|G(U(s, x))-G\left(U\left(s, x-\lambda_{i}^{\xi} \tau\right)\right)\right\| d x d \tau \\
\leq & \mathcal{O}(1) \frac{1}{\theta} \sum_{i=1}^{3} \int_{0}^{\theta} T \cdot V \cdot(G(U(s)) ;(a, b)) \cdot \lambda_{i}^{\xi} \tau d \tau \\
\leq & \mathcal{O}(1) \theta \cdot T \cdot V \cdot(G(U(s)) ;(a, b)) .
\end{aligned}
$$

Consequently we have

$$
\lim _{\theta \rightarrow 0^{+}} \frac{1}{\theta} \int_{a+\theta \hat{\lambda}}^{b-\theta \hat{\lambda}}\left\|\theta G(U(s, x))-U_{\xi}^{2}(\theta, x)\right\| d x=0 .
$$

Then (8) holds for some constant $C_{0}$.

Conversely, suppose mapping $U:[0,+\infty) \rightarrow \mathcal{D}_{\infty}$ is Lipschitz continuous in $L^{1}$-topology and satisfies (7) (8) for $s \in[0,+\infty) \backslash \mathcal{N}$ and $\xi \in \mathbb{R}$, where $\mathcal{N}$ is a set with measure 0 . Given $s \in[0,+\infty) \backslash \mathcal{N}$ and $\varepsilon>0$, we can cover the interval $[a, b]$ with finitely many intervals $\left[a_{i}, b_{i}\right]$ such that

- $\bigcup_{i}\left[a_{i}, b_{i}\right]=[a, b]$ and $\left(a_{i}, b_{i}\right) \bigcap\left(a_{j}, b_{j}\right)=\emptyset$ with $i \neq j$;

- $T . V .\left(U(s) ;\left(a_{i}, b_{i}\right)\right)<\varepsilon ;$

- $\varlimsup_{\theta \rightarrow 0^{+}} \frac{1}{\theta} \int_{a_{i}+\theta \hat{\lambda}}^{b_{i}-\theta \hat{\lambda}}\left\|U(s+\theta, x)-U_{\zeta_{i}}^{T}(\theta, x)\right\| d x \leq C_{0}\left\{T . V .\left(U(s) ;\left(a_{i}, b_{i}\right)\right)\right\}^{2}$ where $\zeta_{i} \doteq\left(a_{i}+b_{i}\right) / 2$.

Set $\xi_{i}=a_{i}$. Then construct a function

$$
U^{*}(t, x) \doteq \begin{cases}U_{\xi_{i}}^{C}(t, x) & \text { if }\left|x-\xi_{i}\right| \leq t \hat{\lambda} \\ U_{\zeta_{i}}^{T}(t, x) & \text { if } a_{i}+t \hat{\lambda}<x<b_{i}-t \hat{\lambda}\end{cases}
$$


for $t$ small enough. Hence

$$
\begin{aligned}
& \varlimsup_{\theta \rightarrow 0^{+}} \frac{1}{\theta} \int_{a+\theta \hat{\lambda}}^{b-\theta \hat{\lambda}}\left\|U(s+\theta)(x)-U^{*}(\theta, x)\right\| d x \\
& \leq \sum_{i} \lim _{\theta \rightarrow 0^{+}} \frac{1}{\theta} \int_{\xi_{i}-\theta \hat{\lambda}}^{\xi_{i}+\theta \hat{\lambda}}\left\|U(s+\theta)(x)-U_{\xi_{i}}^{C}(\theta, x)\right\| d x \\
& \quad+\sum_{i} \varlimsup_{\theta \rightarrow 0^{+}} \frac{1}{\theta} \int_{a_{i}+\theta \hat{\lambda}}^{b_{i}-\theta \hat{\lambda}}\left\|U(s+\theta)(x)-U_{\zeta_{i}}^{T}(\theta, x)\right\| d x \\
& \leq \sum_{i} C_{0}\left\{T \cdot V \cdot\left(U(s) ;\left(a_{i}, b_{i}\right)\right)\right\}^{2} \\
& \leq C_{0} T \cdot V \cdot(U(s) ;(a, b)) \varepsilon .
\end{aligned}
$$

Similarly we have

$$
\varlimsup_{\theta \rightarrow 0^{+}} \frac{1}{\theta} \int_{a+\theta \hat{\lambda}}^{b-\theta \hat{\lambda}}\left\|\mathcal{P}_{s+\theta}\left(U_{0}\right)-U^{*}(\theta, x)\right\| d x \leq C_{0} T . V .\left(\mathcal{P}_{s}\left(U_{0}\right) ;(a, b)\right) \varepsilon .
$$

Therefore,

$$
\begin{gathered}
\varlimsup_{\theta \rightarrow 0^{+}} \frac{1}{\theta} \int_{a+\theta \hat{\lambda}}^{b-\theta \hat{\lambda}}\left\|U(s+\theta)(x)-\mathcal{P}_{s+\theta}\left(U_{0}\right)\right\| d x \\
\leq C_{0}\left\{T . V .(U(s) ;(a, b))+T . V .\left(\mathcal{P}_{s}\left(U_{0}\right) ;(a, b)\right)\right\} \varepsilon .
\end{gathered}
$$

We find $U(s)=\mathcal{P}_{s}\left(U_{0}\right)$ according to the arbitrariness of $(a, b)$ and $\varepsilon$ in the last estimate.

\section{References}

[1] C. Dafermos, L. Hsiao, Hyperbolic systems of balance laws with inhomogeneity and dissipation, Indiana Univ. Math. J. 31 (4) (1982) 471-491.

[2] D. Amadori, L. Gosse, G. Guerra, Global bv entropy solutions and uniqueness for hyperbolic systems of balance laws, Arch. Rational Mech. Anal. 162 (4) (2002) 327-366.

[3] D. Amadori, G. Guerra, Uniqueness and continuous dependence for systems of balance laws with dissipation, Nonlinear Analysis 49 (2002) 987-1014. 
[4] D. Amadori, G. Guerra, Global weak solutions for systems of balance laws, Appl. Math. Lett. 12 (6) (1999) 123-127.

[5] C. Christoforou, Hyperbolic systems of balance laws via vanishing viscosity, Journal of Differential Equations 221 (2) (2006) 470-541.

[6] R. Colombo, G.Guerra, Hyperbolic balance laws with a non local source, Communications in Partial Differential Equations 32 (12) (2007) 19171939.

[7] G. Chen, D. Wagner, Global entropy solutions to exothermically reacting, compressible euler equations, J. Differential Equations 191 (2) (2003) 277322.

[8] K. Hu, Existence of global bv solutions to a model of reacting euler fluid with variable thermodynamics parameters, J. Math.Anal.Appl. 457 (2018) 890-921.

[9] G. Chen, Global solutions to the compressible navier-stokes equations for a reacting mixture, SIAM J. Math. Anal. 23 (3) (1992) 609-634.

[10] G. Chen, D. Hoff, K. Trivisa, Global solutions to a model for exothermically reacting, compressible flows with large discontinuous initial data, Arch. Rational Mech. Anal. 166 (4) (2003) 321-358.

[11] D. Wang, Global solution for the mixture of real compressible reacting flows in combustion, Commun. Pure Appl. Anal 3 (4) (2004) 775-790.

[12] D.Donatelli, K.Trivisa, On the motion of a viscous compressible radiativereacting gas, Commun. Math. Phys. 265 (2) (2006) 463-491.

[13] A. Bressan, T.P.Liu, T.Yang, $\mathrm{L}^{1}$ stability estimates for $n \times n$ conservation laws, Arch. Rational Mech. Anal. 149 (1999) 1-22.

[14] T.P.Liu, T.Yang, $\mathrm{L}^{1}$ stability for $2 \times 2$ systems of hyperbolic conservation laws, J. Amer. Math. Soc. 12 (1999) 729-774. 
[15] A. Bressan, Hyperbolic systems of conservation laws: the one-dimensional cauchy problem, Oxford University Press.

[16] C. Dafermos, Hyperbolic conservation laws in continuum physics, Springer. 